\title{
ECHINOSTOMATOIDEA, NOTOCOTYLATA UND CYCLOCOELIDA (TREMATODA, DIGENEA, REDIOINEI) AUS VÖGELN DES BERLINER TIERPARKS \\ von
}

\author{
KLAUS ODENING
}

Deutsche Akademie der Wissenschaften zu Berlin

Zoologische Forschungsstelle im Berliner Tierpark

(Direktor Prof. Dr. H. Dathe)

INHALTSVERZEICHNIS

A. Großsystematische Vorbemerkungen . . . . 37

Echinochasmidae n. fam. . . . . . . . . 39

Bestimmungsschlüssel zu den Unterfamilien der Echinochasmidae n. fam. . . . . . . . . 40

Bestimmungsschlüssel $z u$ den Gattungen der Echinochasminae, Nephrostomatinae und Microparyphinae . . . . . . . . . 40, 41

B. Spezieller Teil . . . . . . . . . . . . 42

1. Echinoparyphium recurvatum (v. Linstow, 1873) . . . . . . . . . . . . . 42

2. Echinostomatinae gen. sp. . . . . . . . 42

3. Curtuteria grummti n. sp. . . . . . . . 43

4. Petasiger neocomense FunrumanN, 1928 . . 46

5. Echinochasmus dietzevi IsA JČIKov, 1927 . . 46

6. Echinochasmus mergi palaearcticus n. subsp. 47

\section{A. GROSSSYSTEMATISCHE VORBEMERKUNGEN}

Die Subsubclassis Redioinei ODEning, 1960 innerhalb der Unterklasse Digenea (vAN BENEDEN, 1858) wurde in zwei früheren programmatischen Systementwürfen provisorisch, teilweise in Anlehnung an LA RUE (1957), gegliedert (OdEnung 1960, 1961b). Ich halte es heute für angebracht, die in jeder Beziehung bestimmbaren und festumrissenen Trematodengruppen als Ordnungen zu bewerten, wie es z.B. auch in den neueren Systemen der Cestoden der Fall ist. Diese Auffassung hat nicht nur praktische Vorzüge, sondern sie befreit auch die unbestritten einheitlichen Gruppen aus hypothetischen Verbindungen. Ist es doch ein Nachteil der meisten neueren Einteilungsversuche der Digenea, daß phylogenetische Hypothesen in Form von Ordnungen etabliert wurden, die nach Lage der Dinge je nach Auffassung der Autoren recht verschieden zusammengesetzt waren, während die wirklich einheitlichen Gruppen mit den Zwischenkategorien (Unterordnung, Uberfamilie) bedacht wurden. Die Redioinei umfassen nach der neuen Wertung folgende selbständige Ordnungen (alphabetische Reihenfolge):
7. Echinochasmus amphilbolus KoTLÁN, 1922. . 48

8. Echinochasmus beleocephalus (v. Linsrow, 1873 . . . . . . . . . . . . . . 49

9. Echinochasmus coaxatus Dietz, 1909 . . . 51

10. Monilifer spinulosus (RUdolPHI, 1809). . . . 52

11. Mesorchis pseudoechinatus (OLSson, 1876) . 54

12. Episthmium colymbi ŠIGI, 1956 . . . . . 54

13. Ignavia renalis orientalis $\mathrm{n}$. subsp. . . . . 55

14. Psilostomum krevicolle (ChEPLIN, 1829) . . 57

15. Catatropis verrucosa (FröLICH, 1789) . . . 57

16. Paramonostomum alveatum (MEHLIs in CREPLIN, 1846) . . . . . . . . . . . 58

17. Cyclocoelum mutabile (ZEDER, 1800) . . . 59

Literatur . . . . . . . . . . . 59

1. Allocreadiida Odening, 1960

2. Azygiida (LA RuE, 1957) stat. et nom. emend.

3. Clinostomatida (Allison, 1943) stat. et nom. emend.

4. Cyclocoelida (LA RuE, 1957) stat. et nom. emend.

5. Fasciolida (POCHE, 1926) stat. et char. emend.

6. Hemiurida (PociE, 1926) stat. emend.

7. Opisthorchiida (LA RuE, 1957) char. emend.

8. Paramphistomatida (POCHE, 1926) stat. et char. emend.

Die Ordnung Didymozoida (Poche, 1926) ist von den Redioinei auszuschließen, da sie möglicherweise nicht zu den Digenea gehört (siehe BAER \& Joyeux 1961).

Im Folgenden sei die Untergliederung der Ordnungen Fasciolida und Paramphistomatida kurz dargestellt, soweit es für das Verständnis der Einordnung der Arten im speziellen Teil der vorliegenden Arbeit nötig erscheint.

Fasciolida (Poche, 1926) stat. et char. emend.

Echinostomatoidea FAUST, 1929

Balfouriidae Travassos, 1951

Cathaemasiidae FurrmanN, 1928

Cotylotretidae Travassos, 1922

Echinochasmidae (ODHNER, 1910) stat. et char. emend.

Echinocollidae Odening, 1962 (= Saakotrematidae Odening, 1962) 
Echinostomatidae (Looss, 1902)

Ommatobrephidae PocHE, 1926

Philophthalmidae Travassos, 1918

Rhopaliasidae Looss, 1899

PRhytidodidae ODHNER, 1926

Fascioloidea (STHLes \& Goldberger, 1910)

Fasciolidae RAIIIIET, 1895

Campulidae Odmer, 1926

Paramphistomatida (PockE, 1926) stat. et char. emend.

Paramphistomatata (Poche, 1926) Sxrjabin \& Sul'c, 1937

Notocotylata (Poche, 1926) SxRjabin \& SuL'c, 1933

Im speziellen Teil dieser Arbeit werden 14 Species bzw. Subspecies von Echinostomatoidea, 2 Species von Notocotylata und 1 Species von Cyclocoelida beschrieben. Während die Einordnung bei den Notocotylata und Cyclocoelida keine Schwierigkeiten macht, ist bei den Echinostomatoidea eine Auseinandersetzung mit der bisherigen Systematik der Gruppe angebracht. Hier sind zahlreiche Probleme vor allem im Bereich der bisherigen großen Familie Echinostomatidae (sensu lato) sowohl was die Artsystematik als auch was die Begründung der Gattungen und Unterfamilien betrifft noch ungelöst. Die wichtigsten Arbeiten zur. Einteilung der Echinostomatidae verdanken wir Dietz (1910), Odhner (1910), MENDheIM (1940, 1943), BAŠKirova (1941), Skrjabin \& BAŠKIROVA (1956) und YAMAGUTI (1958). Ein Vergleich der neueren Systeme von MendHeim (1943), SxrJabin \& BaŠKIROVA (1956) und Yamaguti (1958) zeigt in vielen Punkten außerordentlich große Unterschiede. Dennoch sind viele Fortschritte erzielt worden, die aber nicht immer in gerader Linie zu erkennen sind.

System der Echinostomatidae nach MENDHEIM (1943):

Nephrostom(at)inae MENDHEDM, 1943

Nephrostomum DiETz, 1909

Patagifer DreTz, 1909

Echinostom(at)inae Looss, 1899

Echinostoma RudoLPH, 1809

Artyfechinostomum LANE, 1915

Echinocirrus MENDHEIM, 1943

Echinoparyphium DETZ, 1909

Euparyphium Dierz, 1909

Hypoderaeum DIETZ, 1909

Moliniella HÜ̈BNER, 1939

Prionosoma DEeTz, 1909

Himasthlinae ODHNER, 1911

Himasthla DiETz, 1909

Acanthoparyphium DIETZ, 1909

Aporchis STOssich, 1905

Cloeophora DIETZ, 1909

Pelmatostomum DIETz, 1909

Echinochasminae ODHNER, 1911

Echinochasmus DiETz, 1909

(Echinochasmus) DIETz, 1909
(Episthmium) LÜHE, 1909

Allechinostomum ODHNER, 1911

Stephanoprora ODHNER, 1902

(Stephanoprora) ODHNER, 1902

(Mesorchis) DiETz, 1909 (Syn. Monilifer DnEtz, 1909)

Velamenophorus MENDHEIM, 1940

Pegosom(at)inae ODHNER, 1911

Pegosomum Ratz, 1903

Paryphostomatinae MENDHEDM, 1943

Paryphostomum DIETz, 1909

Drepanocephalus Duetz, 1909

Chaunocephalinae MENDHEn, 1943

Chaunocephalus DrETz, 1909

Balfouria LEIPER, 1908

Petasigerinae MENDHEIM, 1943

Petasiger DIETZ, 1909

Navicularia MENDHEM, 1943

Microparyphi(i)nae MENDFEIM, 1943

Microparyphium DIETz, 1909

Anhang: Parechinostomum DLETz, 1909.

System der Echinostomatidae nach

SKRJABIN \& BAŠIROVA (1956):

Echinostomatinae ODrner, 1911

Echinostoma RuDoLPHI, 1809

Baschkirovitrema SxrJABI, 1944

Dietziella SkrJabin \& BAŠKIRova, 1956

Drepanocephalus DieTz, 1909

Echinodollfusia SkRJabne \& BAŠKtrova, 1956

Echinoparyphium DIETZ, 1909

Euparyphium DIETZ, 1909

Ignavia TedXeIra de Fremtas, 1948

Longicollia ByChOvSKAjA-PAvLOVSKAJA, 1953

Moliniella HüBNER, 1939

Nephrostomum DIETz, 1909

Parallelotestis BELOPOL'SKA JA 1954

Paryphostomum DiEtz, 1909

Patagifer DrETZ, 1909

Petasiger DreTz, 1909

(Petasiger) DiETz, 1909

(Neopetasiger) BAŠKKRovA, 1941

Prionosoma DIETz, 1909

Allechinostomatinae SUdAruKov, 1950

Allechinostomum ODHNER, 1911

Sobolevistoma SUdARIKov, 1950

Stephanoprora ODHNER, 1902

Chaunocephalinae Travassos, 1922

Chaunocephalus DIETz, 1909

Balfouria LEIPER, 1908

Echinoschasminae ODHNER, 1911

Echinochasmus DETz, 1909

(Echinochasmus) DIETz, 1909

(Monilifer) Dretz, 1909

(Episthmium) LÜHE, 1909

(Episthochasmus) VERMA, 1935

Mesorchis DIETZ, 1909

Saakotrema SKrJABIN \& BAŠKnovA, 1956

Velamenophorus MENDHEIM, 1940

Eurycephalinae SKrJaBIN \& BAŠKIrovA, 1956

Eurycephalus OvčARENKo, 1955

Himasthlinae ODHNER, 1911

Himasthla DIETZ, 1909

Acanthoparyphium DETz, 1909

Aporchis Srossich, 1905 
Artyfechinostomum LANE, 1915

Cloeophora DrETz, 1909

Pelmatostomum DIETZ, 1909

Reptiliotrema BAŠKIROVA, 1941

Hypoderaeinae SkRJABIN \& BAŠKIROVA, 1956

Hypoderaeum DIETz, 1909

Multispinotrema SxRJABIN \& BAŠKIROVA, 1956

Skrjabinophora BAŠxIRovA, 1941

Microparyphiinae MENDHEIM, 1943

Microparyphium DuETz, 1909

Nephroechinostomatinae OŠMarin \& BELous, 1951

Nephroechinostoma OšmaRin \& BELous, 1951

Pegosomatinae OdHNeR, 1911

Pegosomum Ratz, 1903

Sodali(i)nae SkrJabin \& SuL'c, 1937

Sodalis KowaLEwSKa, 1902

System der Echinostomatidae nach

YAMAGUTI ( 1958$)$ :

Balfouriinae YAMAGUT, 1958

Balfouria LEIPER, 1908

Chaunocephalinae Travassos, 1922

Chaunocephalus DiETz, 1909

Cotylotretinae SxrJabin \& SuL'c, 1938

Cotylotretus ODHNER, 1902

Echinochasminae ODHNER, 1910

Allechinostomum ODHNER, 1911

Echinochasmus DIETz, 1909 (Syn. Heterechinostomum ODhNER, 1911; Velamenophorus MendheIM, 1940)

Episthmium LÜHE, 1909 (Syn. Episthochasmus VERMA, 1935)

Microparyphium DIETZ, 1909

Monilifer DnETz, 1909

Patagifer Dietz, 1909

Stephanoprora OdHNER, 1902 (Syn. Mesorchis Dietz, 1909; Sobolevistoma SudARIKov, 1950)

Echinostomatinae (Looss, 1899)

Acanthoparyphium DIETz, 1909

Allopetasiger YAMAGUTI, 1958

Artyfechinostomum LANE, 1915

Baschkirovitrema SkRJABIN, 1944

Cloeophora DieTz, 1909

Drepanocephalus DrETZ, 1909

F.chinoparyphium DrETz, 1909 (Syn. Isoparyphium MENDHEIM, 1940; Moliniella HüBNER, 1939)

Echinostoma RUDOLPHI, 1809

Euparyphium DrExz, 1909

Hypoderaeum DiETz, 1909

Isthmiophora LÜHE, 1909

Neoacanthoparyphium YamaguT, 1958

Nephrostomum DuETz, 1909

Pameileenia Wright \& Smrthens, 1956

Parallelotestis BELOPOL'SKA JA, 1954

Parechinostomum DIETz, 1909

Paryphostomum DIETZ, 1909

Petasiger DiETz, 1909

Prionosoma DIETz, 1909

Protechinostoma BEAvER, 1943

Pseudechinochasmus VERMA, 1936

Pseudechinostomum OdHNER, 1911 (nec Šcupakov, 1936)

Skriabinophora BASKKIROVA, 1941
Testisacculus BhALERAo, 1927 (Syn. Reptiliotrema BAŠKIROVA, 1941)

Himasthlinae ODHNER, 1910

Aporchis Stossich, 1905 (Syn. Macrechinostomum ODHNER, 1911)

Dissurus Verma, 1936

Himasthla DIETZ, 1909

Longicollia Bychovskaja-Pavlovskaja, 1954

Ignaviinae YAMAGUT, 1958

Ignavia TeixeIrA de Frertas, 1948 (Syn. Nephroechinostoma OŠMARIN \& BELous, 1951)

Pegosom(at)inae OdHNER, 1911

Pegosomum Ratz, 1903

Pelmatostom(at)inae Yamaguti, 1958

Pelmatostomum Drerz, 1909

Singhiinae Yamagutx, 1958

Singhia Yamacuti, 1958

Sodaliinae Skrjabin \& Sur'c, 1937

Sodalis Kowal.ewsKı, 1902 (Syn. Scapanosoma LÜhE, 1909; Eurycephalus OvčARENKo, 1955)

Eine Stellungnahme zur Einteilung der Echinostomatoidea in Familien (siehe S. 000) habe ich schon früher veröffentlicht (ODENing 1962a). Eine Veränderung ergibt sich u.a. durch die Zusammenfassung einiger Unterfamilien der Echinostomatidae zur neuen Familie Echinochasmidae. Hinweise auf die Heterogenität der bisherigen großen Familie Echinostomatidae wurden bereits früher gegeben (ODEning 1962d, f). Die neue Familie Echinochasmidae umfaßt alle Formen mit geraden Kragenstachelzahlen und demzufolge dorsaler medianer Unterbrechung der Kragenstachelreihe(n). Typische Unterfamilie sind die Echinochasminae, die zugleich als Ausgangsformen betrachtet werden können. Die übrigen Unterfamilien sind von den Echinochasminae abzuleiten. Die Abgrenzung der neuen Familie Echinochasmidae von denjenigen übrigen Familien der Echinostomatoidea, die im adulten Stadium eine Kopfkragenbestachelung aufweisen, wird durch die folgende Charakteristik erläutert:

Echinostomatidae - Uterus auf den Bereich vor den Testes beschränkt; mit vollständiger Kragenbestachelung ohne dorsale Unterbrechung; dorsaler medianer Spitzenstachel stets vorhanden (ungerade Kragenstachelzahlen).

Echinochasmidae - Uterus auf den Bereich vor den Testes beschränkt; Kragenbestachelung mit dorsaler Unterbrechung oder überhaupt unvollständig, ohne dorsalen medianen Spitzenstachel (gerade Kragenstachelzahlen).

Echinocollidae $=$ Saakotrematidae - Uterus erstreckt sich bis hinter die Testes; Kragenbestachelung mit dorsaler Unterbrechung, ohne dorsalen medianen Spitzenstachel (gerade Kragenstachelzahlen).

Balfouriidae - Uterus erstreckt sich bis hinter die Testes; mit vollständiger Kragenbestachelung ohne dorsale Unterbrechung; dorsaler medianer Spitzenstachel vorhanden (ungerade Kragenstachelzahlen). 
Nicht berücksichtigt sind hierbei die Unterfamilien Parorchiinae LaL, 1936 (Philophthalmidae) und Singhiatrematinae SiмнA, 1962 (Ommatobrephidae). Es folgt eine Übersicht über die Familie Echinochasmidae.

\section{Echinochasminae OdHnER, 1910 (Syn. Allechinostomatinae SuDARrov, 1950) \\ Echinochasmus DrETz, 1909 \\ Allechinostomum ODHNER, 1910 \\ Episthmium LÜHE, 1909 (Syn. Episthochasmus VERMA, 1935) \\ Mesorchis DIETz, 1909 \\ Monilifer DIETZ, 1909 \\ Stephanoprora ODHNER, 1902}

Anhang: Sobolevistoma SudaRIKov, 1950, gen. inquir., möglicherweise mit Mesorchis identisch; Velamenophorus Mendheim, 1940, gen. inquir. - die dieser Gattung zugrundeliegende einzige Art ist möglicherweise mit Mesorchis gracilis (MENDHEIM) identisch, wenn man eine Anomalie in der Ausbildung der Dotterstöcke in Betracht zieht.

Microparyphiinae Mendreim, 1943 (Syn. Nephroechinostomatinae OŠMAfIN \& BELous, 1951; Ignaviinae YAMAGUTI, 1958)

Microparyphium DIETZ, 1909

Ignavia Telxera de Frertas, 1948 (Syn. Nephroechinostoma OŠmarin \& Belous, 1951)

Pelmatostomatinae Yamaguti, 1958

Pelmatostomum DIETZ, 1909

Nephrostomatinae MENDHEIM, 1943

Nephrostomum DETZ, 1909

Patagifer DIETZ, 1909

Sodaliinae Skrjabrn \& Sur'c, 1937 (Syn. Eurycephalinae SkrJaBin \& BašKIROVA, 1956)

Sodalis KowalewsKi, 1902 (Syn. Eurycephalus OvčARENKo, 1955)

Anhang zur Familie: Dissurus Verma, 1936, gen. inquir.

Bestimmungsschlüssel zu den Unterfamilien der Echinochasmidae:

1. Cirrusbeutel reicht nicht hinter den Bauchsaugnapf; Kragen-Randstacheln, soweit vorhanden, einreihig an-

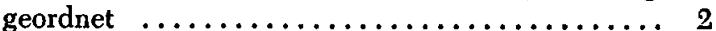

- Vesicula seminalis reicht weit bis hinter den Bauchsaugnapf; Kragen-Randstacheln doppelreihig angeordnet (Zahl der Kragenstacheln 30 bis 34; Kopfkragen biskuitförmig):

Pelmatostomatinae

2. Körperregion vor dem Bauchsaugnapf blattartig verbreitert, mit absgestumpftem Vorderende, an dem anstelle eines Kopfkragens jederseits ein ohrförmiger Lappen ausgebildet ist, an dem nur wenige Eckstacheln sitzen:

Sodaliinae

- Körper vor dem Bauchsaugnapf nicht verbreitert. . 3

3. Kopfkragen nieren- oder herzförmig (Zahl der Kragenstacheln 20 bis 34$) \ldots \ldots \ldots \ldots \ldots \ldots \ldots 4$

- Kopfkragen biskuitförmig (zweilappig), mit dorsaler Einbuchtung oder dorsalem Einschnitt (Zahl der Kragenstacheln 32 bis 64 ):

Nephrostomatinae
4. Kopfkragen gut entwickelt, dorsale Unterbrechung der Kragenstachelreihe nur kurz (Zahl der Kragenstacheln 20, 22, 24 bzw. 26 oder 30, 32 bzw. 34); Mundsaugnapf meist nicht breiter als die Hälfte des Kopfkragens:

Echinochasminae

- Kopfkragen schwach entwickelt, mit langer dorsaler Unterbrechung der Kragenstachelreihe (Zahl der Kragenstacheln 20, 22 bzw. 24); Mundsaugnapf kräftig entwickelt, stets mindestens so breit wie die Hälfte des Kopfkragens:

Microparyphiinae

Bestimmungsschlüssel zu den Gattungen der Echinochasminae:

1. Dotterstöcke vor den Bauchsaugnapf reichend und dort zusammenfließend:

Episthmium

- Dotterstöcke den Bauchsaugnapf nach vorn nicht überschreitend $\ldots \ldots \ldots \ldots \ldots \ldots \ldots \ldots \ldots$.

2. Dotterstöcke die Höhe des Vorderrandes des vorderen Testis nach vorn nicht überschreitend ........ 3

- Dotterstöcke reichen über den vorderen Testis nach vorn hinaus $\ldots \ldots \ldots \ldots \ldots \ldots \ldots \ldots \ldots \ldots$

3. Parasiten von Reptilien:

Stephanoprora

- Parasiten von Vögeln oder Säugetieren ....... 4

4. Körper sehr langgestreckt und schmal, Körperbereich hinter den Testes lang; Testes meist längsoval (selten rundlich):

Mesorchis

- Körper gedrungener, Körperbereich hinter den Testes meist nicht so lang; vorderer Testis breitgestreckt, hinterer Testis herzförmig mit nach hinten gerichteter Spitze:

5. Parasiten von Vögeln oder Säugetieren:

Monilifer

- Parasiten von Reptilien:

Echinochasmus

Allechinostomum

Während die Gattung Episthmium sich gut von den übrigen Gattungen der Echinochasminae unterscheiden läßt, ist die Genusabgrenzung bei letztgenannten noch nicht befriedigend. So gibt es kaum hinreichende morphologische Unterschiede, die eine Trennung von Echinochasmus und Allechinostomum ermöglichen würden. Ein „Stachelvelum“ ist bei Vertretern mehrerer Gattungen nachweisbar; es handelt sich dabei um eine schwimmhautartige cuticulare Verbindung zwischenden einzelnen Kragenstacheln. Diesem Merkmal dürfte kaum systematischer Wert zukommen. Weiter ist die Abgrenzung von Mesorchis und Monilifer problematisch (siehe S. 00). Monilifer kann man als intermediär zwischen Mesorchis und Echinochasmus ansehen (wobei aber nach Zugrundelegung des bei der Einteilung der Unterfamilie üblichen Prinzips der Ausdehnung der Dotterstöcke nach vorn eine nähere Verwandtschaft zu Mesorchis angenommen werden müßte). Innerhalb der Gattung Echinochasmus gibt es eine Artengruppe mit den ab- 
weichenden Kragenstachelzahlen 30, 32, 34. Diese ostasiatische Gruppe scheint auch durch ihren Zyklus (Frösche und Schnecken als Hilfswirte) von den übrigen Echinochasmus-Arten (Fische als Hilfswirte) abzuweichen. Hier scheint eine natürliche Einteilung in zwei Untergattungen vorgezeichnet zu sein. Alle diese hier aufgeworfenen Fragen können nur befriedigend beantwortet werden, wenn Entwicklung und Cercarientypen in größerem Umfang als bisher bekannt sein werden. Ein weiteres Problem ist die Abgrenzung von Mesorchis und Stephanoprora. Die Genera inquirenda Sobolevistoma und Velamenophorus wurden absichtlich im Bestimmungsschlüssel nicht berücksichtigt.

Bestimmungsschlüssel zu den Gattungen der Nephrostomatinae:

Kopfkragen biskuitförmig mit flacher dorsaler Einbuchtung (Zahl der Kragenstacheln 32 bis 50 ):

Nephrostomum

Kopfkragen biskuitförmig mit tiefem dorsalem Einschnitt (Zahl der Kragenstacheln 42 bis 64):

Patagifer

Bestimmungsschlüssel zu den Gattungen der Microparyphiinae:

Körper länglich-lanzettförmig, Bauchsaugnapf im 2. Körperviertel; Darmparasiten:

Microparyphium

Körper sehr langgestreckt, Bauchsaugnapf im 1. Körperviertel; Nierenparasiten:

Ignavia

Noch einige Bemerkungen zur Einteilung der Echinostomatidae char. emend. (d.h. abzüglich der als eigene Familien eliminierten Gruppen Echinochasmidae, Cotylotretidae, Balfouriidae, Echinocollidae = Saakotrematidae), die jetzt also nur noch Formen mit vollständiger Kragenbestachelung ohne dorsale Unterbrechung und mit ungeraden Kragenstachelzahlen umfassen (Uterus vor den Testes). Es verbleiben innerhalb der Echinostomatidae die Unterfamilien Echinostomatinae, Paryphostomatinae, Petasigerinae, Hypoderaeinae, Himasthlinae, Pegosomatinae, Singhiinae (?) und Chaunocephalinae. Während die Chaunocephalinae (einzige Gattung Chaunocephalus), Pegosomatinae (einzige Gattung Pegosomum), Himasthlinae (Himasthla, Curtuteria, Caballerotrema, Acanthoparyphium, Aporchis, Cloeophora, Artyfechinostomum $\mathrm{P}=$ Testisacculus $=$ Reptiliotrema, ? Pseudechinostomum gen. inquir.) und vielleicht auch die Hypoderaeinae (Hypoderaeum, Multispinotrema, Skrjabinophora) und die Singhiinae (einzige Gattung Singhia; Originalbeschreibung war mir nicht zugänglich) gut umgrenzte Gruppen darstellen, ist die Abgrenzung der Echinostomatinae, Paryphostomatinae und Petasigerinae problematisch. Eine eingehende klärende Revision ist nach der Literatur kaum möglich. Mit hoher Wahrscheinlichkeit bietet jedoch die Anordnung der Kragenstacheln in Verbindung mit anderen Merkmalen die Möglichkeit einer natürlichen Gruppierung (z.B. einreihige oder doppelreihige Anordnung, Vorhandensein oder Fehlen von versetzt stehenden oder durch ihre Größe hervortretenden Eckstacheln; Größenverhältnisse von Stachelreihen oder -gruppen). Auf diese Dinge sollte künftig bei Neu- und Nachbeschreibungen verstärkt geachtet werden (Lebendbeobachtungen sind hier besonders zu empfehlen, da bei fixiertem Material oft Beschädigungen der Kragenbestachelung vorkommen). Auch die Beschaffenheit der Vesicula seminalis (geteilt oder ungeteilt) und des Cirrus (bestachelt oder nicht) müßte untersucht werden. Es ist unter diesen Gesichtspunkten möglich, daß einige der bisherigen großen Gattungen (z.B. Echinostoma und Echinoparyphium) weiter aufgeteilt werden können. Eine Gruppierung nach der Kragenstachelanordnung könnte provisorisch folgendermaßen geschehen:

a) Echinostomatinae - Kragenstacheln doppelreihig angeordnet (Echinostoma, Echinoparyphium = Parechinostomum, Echinodollfusia, Euparyphium $=$ Isthmiophora $P=$ Echinocirrus, Moliniella, Neoacanthoparyphium $=$ Allopetasiger, Parallelotestis $=$ Proechinocephalus Srivastava, 1958, Prionosoma).

b) Paryphostomatinae - Kragenstacheln einreihig angeordnet (Paryphostomum, Baschkirovitrema, Dietziella, Drepanocephalus, Longicollia, Pameileenia, Petasiger $=$ Navicularia $=$ Neopetasiger $)$.

Es muß jedoch betont werden, daß die Deutung der Ein- oder Doppelreihigkeit der Kragenstachelanordnung nicht nur anhand der Literatur schwierig ist, sondern auch oft genug am Tier selbst. Bei Cercarie, Metacercarie und Marita muß Ubereinstimmung in der Anordnung der Kragenstacheln vorliegen (sekundäre Verwischung der ursprünglichen einreihigen Anordnung bei den Maritae von Paryphostomum und Petasiger!). Die Abgrenzung der großen Gattungen Echinostoma und Echinoparyphium befriedigt immer noch nicht (siehe Macko \& OdEning 1963). Auch die Trennung von Echinostoma bzw. Echinoparyphium und Moliniella (= Isoparyphium) ist problematisch. Pseudechinochasmus is als gen. inquir. zu behandeln. Es ist sehr wahrscheinlich, daß dem Studium der Entwicklung des Exkretionssystems bei den Cercarien der Echinostomatoidea im Hinblick auf die Klärung der Verwandtschaftsverhältnisse eine große Bedeutung zukommt (vgl. Odening 1962d). 
B. SPEZIELLER TEIL

I. Fasciolida (Poche, 1926) stat. et char. emend.

Echinostomatoidea Faust, 1929

Echinostomatidae (Looss, 1902) char. emend.

Echinostomatinae Looss, 1899

\section{Echinoparyphium recurvatum (v. Linstow,} 1873)

Wirt/Herkunft: Melanitta fusca (L.), 1 \% / in BerlinHohenschönhausen aufgefundener Wintergast, Sektion am 7.2.1963.

Lokalisation: Dünndarm.

Präparat-Nr.: kT 15/13-15 (90 Exemplare).

Beschreibung (vgl. Abb. 1): Cuticula bestachelt; Körperlänge $2-3,6 \mathrm{~mm}$, maximale Körperbreite $0,3-0,8 \mathrm{~mm}$; Kopfkragen mit $(4+37+4)$ Kragenstacheln, von denen die 4 Eckstacheln jeder Seite wesentlich größer sind als die Randstacheln; alle Kragenstacheln in 2 Reihen paarweise angeordnet (Stacheln der aboralen Reihe größer als die der oralen); Länge der Eckstacheln 55-58 $\mu \mathrm{m}$, Länge der aboralen Randstacheln 48-51 $\mu \mathrm{m}$, der oralen Randstacheln 46-49 $\mu \mathrm{m}$; die Exemplare entsprechen in ihren Körperproportionen der "typischen“ (MACKo \& ODE-

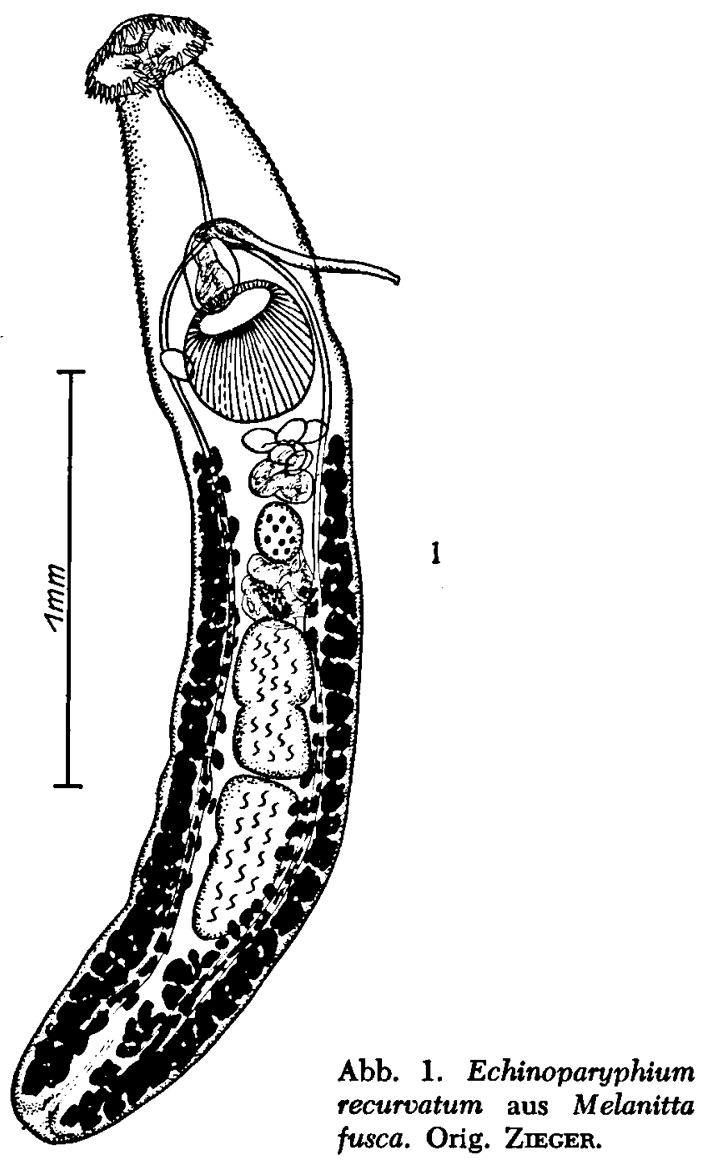

NING 1963; siehe auch Odening 1961a und im Druck b) E. recurvatum; Zahl der Eier meist zwischen 5 und 20; Eigröße $79-114 \times 40-63 \mu \mathrm{m}$.

Bemerkungen. Es sind 3 sehr ähnliche Echinoparyphium-Arten beschrieben: E. recurvatum, E. baculus DieTz, 1909 und E. mordwilkoi SKrJabin, 1915. Auch E. phasianinum GaGarIN, 1954 könnte hier noch angereiht werden. Auch innerhalb des als $E$. recurvatum bezeichneten Formenkomplexes ist die Problematik einer möglichen Heterogenität noch nicht geklärt (siehe Odening 1962f, Macko \& Odening 1963), vor allem im Hinblick auf das Auftreten der Altrices in verschiedenen Schneckenfamilien. ReIMER (1962) fand $E$. recurvatum in Melanitta fusca, $M$. nigra und Anas platyrhynchos auf der Insel Hiddensee, ich selbst fand sie in Larus ridibundus und Strix aluco (siehe Odening 1961a und im Druck b). Bezubix (1956b) und SulgostowsKa $(1958,1960 \mathrm{a})$ meldeten die Art aus Polen.

\section{Echinostomatinae gen. sp.}

Wirt/Herkunft: Phoenicopterus ruber L. / importiert aus Kuba am 8.8.1962, Sektion am 7.9.1962 (nur 1 Tier aus Kuba untersucht).

Lokalisation: Dünndarm.

Präparat-Nr.: kT 13/13-14 (5 Exemplare).

Beschreibung (vgl. Tabelle 1 und Abb. 2): Cuticula im Vorderkörper bestachelt; Kopfkragen nierenförmig, mit $(4+29+4)$ Kragenstacheln, von denen die jederseits 4 Eckstacheln größer sind als die in 2 Reihen angeordneten Randstacheln, von welchen diejenigen der aboralen Reihe etwas größer sind als die der oralen Reihe.

Bemerkungen. Es liegen nur präadulte Exemplare vor. Sie gehören offenbar zu einer Echinostoma- oder Echinoparyphium-Art.

TABelle 1. Echinostomatinae gen.sp. aus Phoenicopterus ruber, Maße der 5 Exemplare in $\mathrm{mm}$

\begin{tabular}{|l|l|l|l|l|l|}
\hline $\begin{array}{l}\text { Körperlänge } \\
\text { max. Körperbreite }\end{array}$ & 0,77 & 1,37 & 1,20 & 1,33 & 1,42 \\
Kopfkragen & & 0,37 & 0,29 & 0,34 & 0,31 \\
$\quad$ Länge & 0,069 & 0,104 & 0,100 & 0,110 & 0,086 \\
$\begin{array}{l}\text { Breite } \\
\text { Mundsaugnapf } \\
\text { Länge }\end{array}$ & 0,131 & 0,193 & 0,161 & 0,183 & 0,195 \\
$\begin{array}{l}\text { Breite } \\
\text { Bauchsaugnapf } \\
\text { Länge }\end{array}$ & 0,070 & 0,097 & 0,088 & 0,084 & 0,093 \\
Breite & 0,063 & 0,079 & 0,072 & 0,077 & 0,083 \\
Pharynx & 0,152 & 0,231 & 0,200 & 0,193 & 0,207 \\
$\quad$ Länge & 0,135 & 0,231 & 0,179 & 0,190 & 0,192 \\
Breite & 0,051 & 0,063 & 0,060 & 0,058 & 0,065 \\
& 0,048 & 0,056 & 0,053 & 0,051 & 0,060 \\
\hline
\end{tabular}



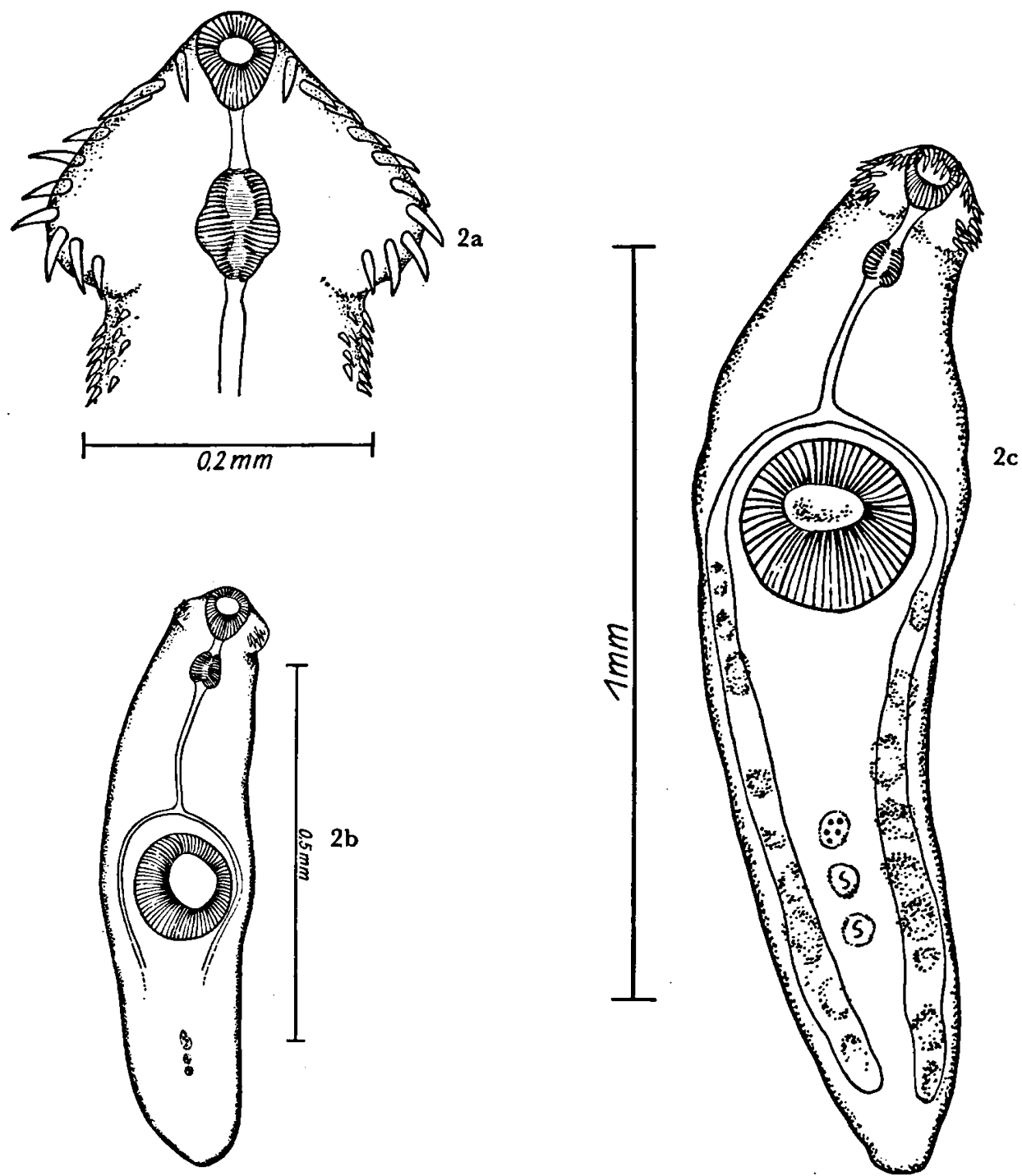

Abb. 2. Echinostomatinae gen. sp. aus Phoenicopterus ruber. a Kopfkragen, b und c Praeadulti verschiedener Entwicklungsstufe. Orig. ZIEGER.

Himasthlinae ODHNER, 1910

\section{Curtuteria grummti n. sp.}

Wirt/Herkunft: Somateria mollissima (L.), 1 \% / von der Insel Hiddensee, Sektion am 6.2.1963.

Lokalisation: Dünndarm.

Präparat-Nr.: kT 15/26-27 (33 Exemplare).

Beschreibung (vgl. Tabelle 2 und Abb. 3): Cuticula vor allem im Vorderkörper mit spitzdachziegelartigen Schuppen besetzt; Körper im Umriß flaschen- oder keulenförmig, $1-3,2 \mathrm{~mm}$ lang, größte Körperbreite $0,6-0,9 \mathrm{~mm}$ (zwischen Ovarium und Bauchsaugnapf oder weiter hinten); Kopfkragen mit $(3+23+3)$ Kragenstacheln, Eckstacheln kleiner als Randstacheln, letztere in einer Reihe angeordnet; Länge des klein- sten, inneren Eckstachels 26-33 $\mu \mathrm{m}$, Länge der übrigen Kragenstacheln 40-65 $\mu \mathrm{m}$ (maximale Breite an der Basis 9-19 $\mu \mathrm{m}$ ); Dotterstöcke meist bis zum Hinterrand des Bauchsaugnapfs reichend; Ovarium kugelig bis queroval, glattrandig, median oder submedian gelegen; Cirrusbeutel sehr lang, weit hinter den Bauchsaugnapf reichend, mit stark entwickelter Vesicula seminalis, Pars prostatica und fein bestacheltem Cirrus; Vorderer Testis meist breitgestreckt oval, trapez- oder wurstförmig, seltener rundlich oder länglich-elliptisch; hinterer Testis meist annähernd dreieckig mit nach hinten gerichteter Spitze, seltener rundlich oder länglich-elliptisch, bei einigen Exemplaren stark verkleinert bis zur völligen Reduktion (bisweilen sind sogar beide Testes völlig reduziert); Eigröße $79-92 \times 48-56 \mu \mathrm{m}$. 


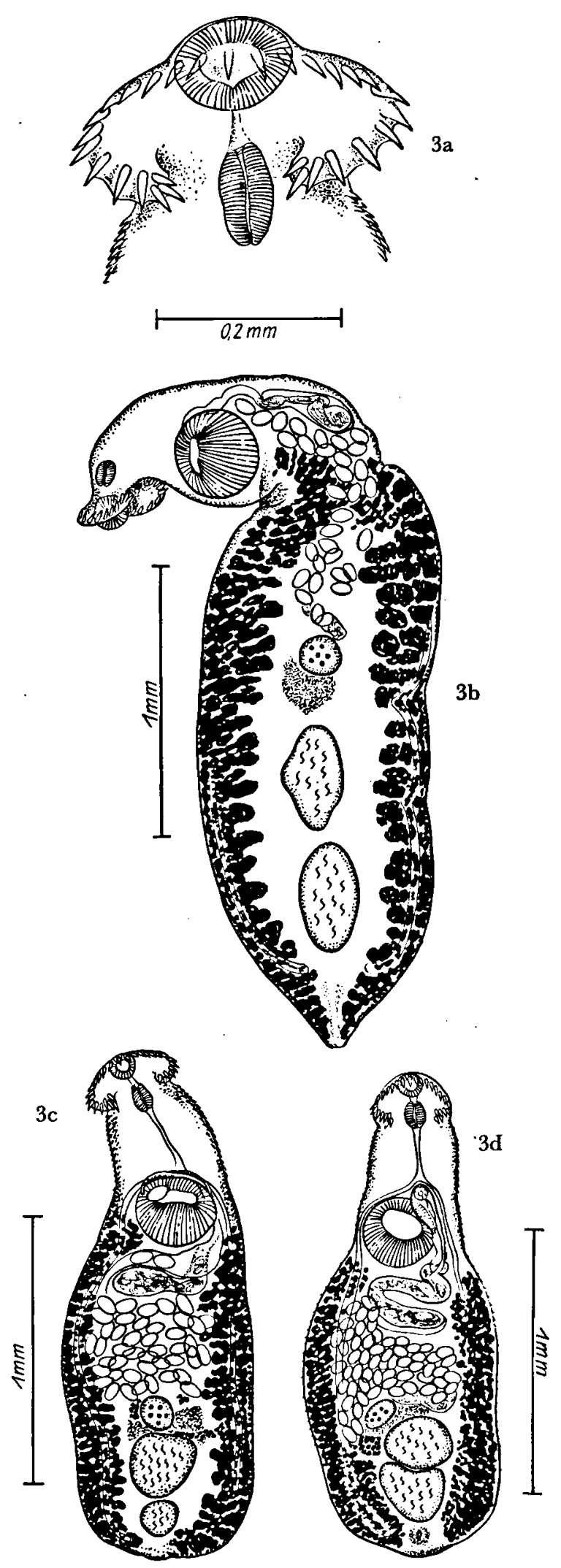

Typus: Das der Abb. 3d zugrundeliegende Exemplar. Helminthensammlung Zoologische Forschungsstelle im Berliner Tierpark Nr. kT 15/27.

Bemerkungen. Die neue Art widme ich dem Kurator für Vögel des Berliner Tierparks, Herrn W. Grummt, dem ich für seine ständige wertvolle Unterstützung durch Zweisung von Material herzlich danken möchte. - Das Genus Curtuteria ReImer, 1963 wurde erst kürzlich entdeckt in der ihm zugrundeliegenden typischen Art C. numenii REIMER, 1963, die ebenfalls von der Insel Hiddensee bekannt wurde, und zwar aus Numenius phaeopus. Die in Somateria mollissima von der Insel Hiddensee gefundenen Exemplare gehören nun zweifellos ebenfalls zur Gattung Curtuteria, lassen sich aber der Art C. numenii nicht zuordnen (siehe untenstehenden Bestimmungsschlüssel). REIMER wies bereits 1962 darauf hin, daß seine neue Art Merkmale der Gattungen Himasthla (Anordnung der Kragenstacheln) und Acanthoparyphium (Körperproportionen) in sich vereinige. Die Gattung Curtuteria ist völlig zu Recht aufgestellt worden, da ihre Vertreter in keine der bisherigen Gattungen der Himasthlinae eingeordnet werden können.

Bestimmungsschlüssel für die Arten des Genus Curtuteria ReIMER, 1963 (Zugleich Differentialdiagnose für C. grummti n. sp.):

Körper im Umriß flaschen- oder keulenförmig, hinter dem Bauchsaugnapf ziemlich gleichmäßig deutlich verbreitert; Körperbreite 0,6-0,9 mm; Bauchsaugnapf 0,213-0,330 $\mathrm{mm}$; Pharynx 0,088-0,116 $\times 0,055-0,079 \mathrm{~mm}$; Randstacheln 40-65 $\mu \mathrm{m}$ lang; Parasiten von Anseriformes: Curtuteria grummti n. sp.

Körper gleichmäBig oblong, hinter dem Bauchsaugnapf kaum merklich verbreitert; Körperbreite 0,29-0,35 mm; Bauchsaugnapf 0,170-0,195 mm; Pharynx 0,058-0,072 $\times$ 0,039-0,051 mm; Randstacheln 28-40 $\mu \mathrm{m}$ lang; Parasiten von Limicolae:

Curtuteria numenii REIMER, 1963

Die Gattung Curtuteria kann auf Grund der Auffindung der zweiten Art folgendermaßen definiert werden: „Echinostomatidae (sensu stricto), Himasthlinae; Körper im Umriß flaschen- oder keulenförmig oder gleichmäßig oblong, hinter dem Bauchsaugnapf deutlich ziemlich gleichmäßig verbreitert oder kaum verbreitert; mit bestachelter Cuticula; Kopfkragen

Abb. 3. Curtuteria grummti n. sp. aus Somateria mollissima. a Kopfkragen, b größtes Exemplar, c Exemplar mit anomal verkleinertem hinterem Testis, d Typus. Orig. ZIEGER. 
nierenförmig ohne ausgeprägte ventrale Verbindungskante, mit einreihiger Anordnung der Kragenstacheln und zwei aus kleineren Stacheln bestehenden Gruppen von Eckstacheln; Uterus höchstens etwa die vordere Hälfte des Hinterkörpers einnehmend, mit bis zu etwa 80 Eiern; Testes median hintereinander in der hinteren Hälfte des Hinterkörpers; Dotterstöcke an den Körperseiten, das Ovarium weit nach vorn überschreitend, aber nicht über den Bauchsaugnapf nach vorn hinausgehend, ohne Zusammenfließen hinter den Testes; Cirrusbeutel sehr lang, weit hinter den Bauchsaugnapf reichend, mit ungeteilter, bisweilen wenig gewundener Vesicula seminalis, mit Pars prostatica und fein bestacheltem Cirrus.“ Die Stellung innerhalb der Unterfamilie Himasthlinae wird durch den folgenden Schlüssel erläutert.

Bestimmungsschlüssel zu den Gattungen der Unterfamilie Himasthlinae:

1. Dotterstöcke vor dem Bereich der im Körperhinterende liegenden Testes; Eier mit Filament (Zahl der Kragenstacheln $31,43,55$ bzw. 57):

Aporchis Stossich, 1905

- Dotterstöcke auch neben und hinter oder überhaupt nur hinter den Testes; Eier ohne Filament ..... 2
2. Kragenstacheln zweireihig angeordnet (Zahl der Kragenstacheln 41); Testes tief fingerförmig gelappt:

Artyfechinostomum LANE, 1915 (? = Testisacculus BHALERAO, $1927=$ Reptiliotrema BAŠKIROVA, 1941)

- Kragenstacheln einreihig angeordnet; Testes überwiegend ganzrandig, selten flach gelappt ..... 3

3. Dotterstöcke auf den Bereich hinter den Testes beschränkt (Zahl der Kragenstacheln 23 bzw. 29): Cloeophora DiETz, 1909

- Dotterstöcke auch neben den Testes und vor dem Bereich der Testes .................. 4

4. Besondere Eckstachelgruppen sind nicht ausgebildet (Zahl der Kragenstacheln 23); Uterusbereich höchstens gleich der Hälfte der Länge des Hinterkörpers: Acanthoparyphium DiErz, 1909

- Je eine durch größere Kragenstacheln abweichende Eckstachelgruppe vorhanden (Zahl der Kragenstacheln 29):

Caballerotrema PRudhoe, 1960

- Je eine durch kleinere Kragenstacheln abweichende Eckstachelgruppe vorhanden $\ldots \ldots \ldots \ldots \ldots \ldots 5$

5. Uterusbereich höchstens gleich der Hälfte der Länge des Hinterkörpers, maximale Eizahlen bis 80; Testes in der hinteren Hälfte des Hinterkörpers (Zahl der Kragenstacheln 29):

Curtuteria REIMER, 1963

TABELle 2. Curtuteria grummti n. sp. aus Somateria mollissima, Maße von 10 Exemplaren in mm.

\begin{tabular}{|c|c|c|c|c|c|c|c|c|c|c|}
\hline \multirow{3}{*}{$\begin{array}{l}\text { Körperlänge } \\
\text { max. Körperbreite } \\
\text { Kopfkragen }\end{array}$} & \multicolumn{7}{|c|}{ Exemplare mit normal ausgebildeten Testes } & \multicolumn{3}{|c|}{$\begin{array}{l}\text { Testes anomal } \\
\text { entwickelt }\end{array}$} \\
\hline & 2,56 & 1,68 & 1,75 & 1,58 & 2,05 & 1,64 & 1,70 & 1,49 & 1,47 & 1,82 \\
\hline & 0,85 & 0,75 & 0,70 & 0,61 & 0,78 & 0,70 & 0,68 & 0,65 & 0,84 & 0,77 \\
\hline Länge & 0,184 & 0,110 & 0,169 & 0,132 & 0,184 & 0,213 & 0,176 & 0,176 & 0,139 & 0,147 \\
\hline Breite & 0,385 & 0,308 & 0,330 & 0,264 & 0,382 & 0,338 & 0,308 & 0,323 & 0,352 & 0,308 \\
\hline \multicolumn{11}{|l|}{ Mundsaugnapf } \\
\hline Länge & 0,113 & 0,095 & 0,106 & 0,081 & 0,106 & 0,099 & 0,109 & 0,109 & 0,092 & 0,097 \\
\hline Breite & 0,125 & 0,109 & 0,125 & 0,093 & 0,120 & 0,141 & 0,120 & 0,114 & 0,123 & 0,109 \\
\hline \multicolumn{11}{|l|}{ Pharynx } \\
\hline Länge & 0,116 & 0,102 & 0,097 & 0,088 & 0,106 & 0,106 & 0,114 & 0,097 & 0,114 & 0,099 \\
\hline Breite & 0,069 & 0,076 & 0,070 & 0,069 & 0,062 & 0,055 & 0,065 & 0,079 & 0,056 & 0,065 \\
\hline \multicolumn{11}{|l|}{ Bauchsaugnapf } \\
\hline Länge & 0,308 & 0,294 & 0,264 & 0,224 & 0,272 & 0,250 & 0,279 & 0,242 & 0,213 & 0,316 \\
\hline Breite & 0,330 & 0,257 & 0,286 & 0,250 & 0,323 & 0,257 & 0,268 & 0,286 & 0,279 & 0,316 \\
\hline \multicolumn{11}{|l|}{ Cirrusbeutel- } \\
\hline Länge & 0,93 & 0,42 & 0,47 & 0,68 & 0,65 & 0,61 & 0,63 & 0,58 & 0,91 & 0,70 \\
\hline \multicolumn{11}{|l|}{ Ovarium } \\
\hline Länge & 0,128 & 0,086 & 0,107 & 0,083 & 0,107 & 0,093 & 0,121 & 0,093 & 0,086 & 0,135 \\
\hline Breite & 0,159 & 0,138 & 0,145 & 0,114 & 0,135 & 0,138 & 0,152 & 0,117 & 0,138 & 0,159 \\
\hline \multicolumn{11}{|l|}{ Vorderer Testis } \\
\hline Länge & 0,220 & 0,147 & 0,169 & 0,110 & 0,198 & 0,154 & 0,147 & 0,110 & 0,117 & fehlt! \\
\hline Breite & 0,375 & 0,286 & 0,330 & 0,323 & 0,268 & 0,279 & 0,367 & 0,154 & 0,235 & \\
\hline \multicolumn{11}{|l|}{ Hinterer Testis } \\
\hline Länge & 0,308 & 0,198 & 0,220 & 0,213 & 0,220 & 0,235 & 0,272 & 0,110 & 0,184 & 0,264 \\
\hline Breite & 0,330 & 0,235 & 0,301 & 0,220 & 0,356 & 0,213 & 0,301 & 0,139 & 0,191 & 0,246 \\
\hline
\end{tabular}


- Uterusbereich länger als die Hälfte des Hinterkörpers, maximale Eizahlen bis über 200; Testes im letzten Viertel oder Drittel des Hinterkörpers (Zahl der Kragenstacheln 25, 27, 29, 31, 33 bzw. 37):

Himasthla DHETz, 1909

Paryphostomatinae MENDHEIM, 1943

\section{Petasiger neocomense FuHRMann, 1928.}

Wirt/Herkunft: Podiceps c. cristatus (L.) / ein aus Berlin-Rummelsburg eingeliefertes Tier, Sektion am 24.5.1962 (von Mitte 1959 bis 15.4.1963 waren insgesamt 10 Haubentaucher aus dem Gebiet von Berlin untersucht worden).

Lokalisation: Dünndarm.

Präparat-Nr.: kT 11/38 (1 Exemplar).

Beschreibung (vgl. Abb. 4): Cuticula bestachelt; Körper spindelförmig, 1,69 mm lang bei einer maximalen Breite von 0,34 mm; Kopfkragen mit $(4+11+4)$ Kragenstacheln, die hier doppelreihig angeordnet erscheinen (sekundär?), Eckstacheln größer als Randstacheln; Eigröße $79-90 \times 48-59 \mu \mathrm{m}$.

Bemerkungen. Die Art wurde von Mendheim (1943), Macko (1959, 1961/62) und Sulcostowska (1960a) beim gleichen Wirt angetroffen. Sie kommt auch in Podiceps ruficollis des gleichen Raumes vor (ODENING im Druck a). Diskussion der Petasiger-Arten aus Podicipedidae siehe OdEning (1962e).

Echinochasmidae (ODHNER, 1910) stat. et char. emend.

Echinochasminae OdHNER, 1910

\section{Echinochasmus dietzevi IsAJčIxov, 1927}

Wirt/Herkunft: Mergus m. merganser L. / ein aus der DDR eingeliefertes Exemplar, Sektion am 18.12. 1962 (von Mitte 1959 bis 15.4.1963 nur 2 Gänsesäger untersucht).

Lokalisation: Dünndarm.

Präparat-Nr.: kT 13/26-27, 32 (12 Exemplare).

Beschreibung (vgl. Tabelle 3 und Abb. 5): Cuticula bestachelt; Körper mit deutlich verschmälertem Halsteil, 0,916 - 1,246 mm lang bei einer maximalen Breite (meist im Bereich des Ovariums oder des vorderen Testis) von 0,345 - 0,455 mm; Kopfkragen mit 20 Kragenstacheln; die Dotterstöcke reichen bis zum Hinterrand oder bis zur Mitte des Bauchsaugnapfs; Praepharynx manchmal fast so lang wie Pharynx; Uterusbereich sehr kurz; Testes meist in der vorderen Hälfte des Hinterkörpers (Hinterkörper = Bereich hinter dem Bauchsaugnapf); Eigröße 72 $107 \times 48-69 \mu \mathrm{m}$.

Bemerkungen. Reimer (1962) fand die Art in Podi-

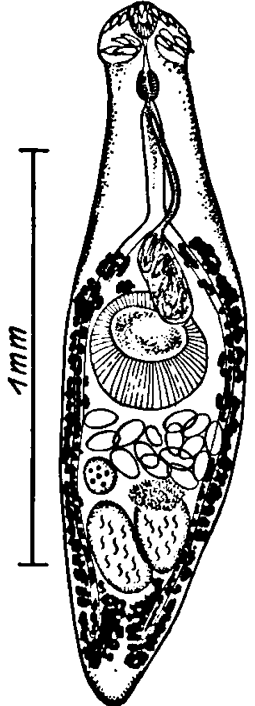

Abb. 4. Petasiger neocomense aus Podiceps cristatus. Orig. ZTEGER.

ceps griseigena, $P$. cristatus, Clangula hyemalis und Mergus serrator, Insel Hiddensee. Weitere Funde wurden aus Polen (BezuBir 1956a) und der Slowakei (Macko 1959, 1961/62) gemeldet. Die Species ist offenbar gegenüber dem Endwirt wenig spezifisch; sie wurde außer aus Podicipediformes und Anseriformes auch aus Lari gemeldet (siehe BychovsKajAPavlovskaja 1962). Eine sehr ähnliche Art ist $E$. donaldsoni BeAver, 1941 aus Nordamerika. Auch $E$. magniovatus (STUNKard \& Haviland, 1924), Nordamerika, läßt sich hier anreihen. Meine Exemplare stimmen in der Körperform gut mit $E$. dietzevi überein; dagegen sind die Maße von Bauchsaugnapf, Pharynx und Kopfkragen kleiner und entsprechen darin eher den beiden nordamerikanischen Arten.

TABelle 3. Echinochasmus dietzevi aus Mergus merganser, Maße von 5 Exemplaren in mm.

\begin{tabular}{|c|c|c|c|c|c|}
\hline Körperläng & 0,982 & 1,173 & 1,100 & 0,916 & 1,004 \\
\hline $\begin{array}{l}\text { max. Körperbreite } \\
\text { Kopfkragen }\end{array}$ & 0,367 & 0,440 & 0,396 & 0,418 & 0,426 \\
\hline Länge & 0,169 & 0.139 & 0.147 & 0,169 & 0.154 \\
\hline Breite & 0,198 & 0,184 & 0,191 & 0,184 & 0,213 \\
\hline Mundsaugnapf & & & & & \\
\hline Länge & 0,062 & 0,066 & 0,072 & 0,069 & 0,066 \\
\hline Breite & 0,076 & 0,079 & 0,086 & 0,079 & 0,076 \\
\hline Bauchsaugnapf & & & & & \\
\hline Länge & 0,086 & 0,072 & 0,079 & 0,114 & 0,131 \\
\hline Breite & 0,121 & 0,097 & 0,086 & 0,117 & 0,121 \\
\hline Pharynx & & & & & \\
\hline Länge & 0,055 & 0,059 & 0,062 & 0,086 & 0,069 \\
\hline Breite & 0,048 & 0,048 & 0,052 & 0,059 & 0,069 \\
\hline
\end{tabular}



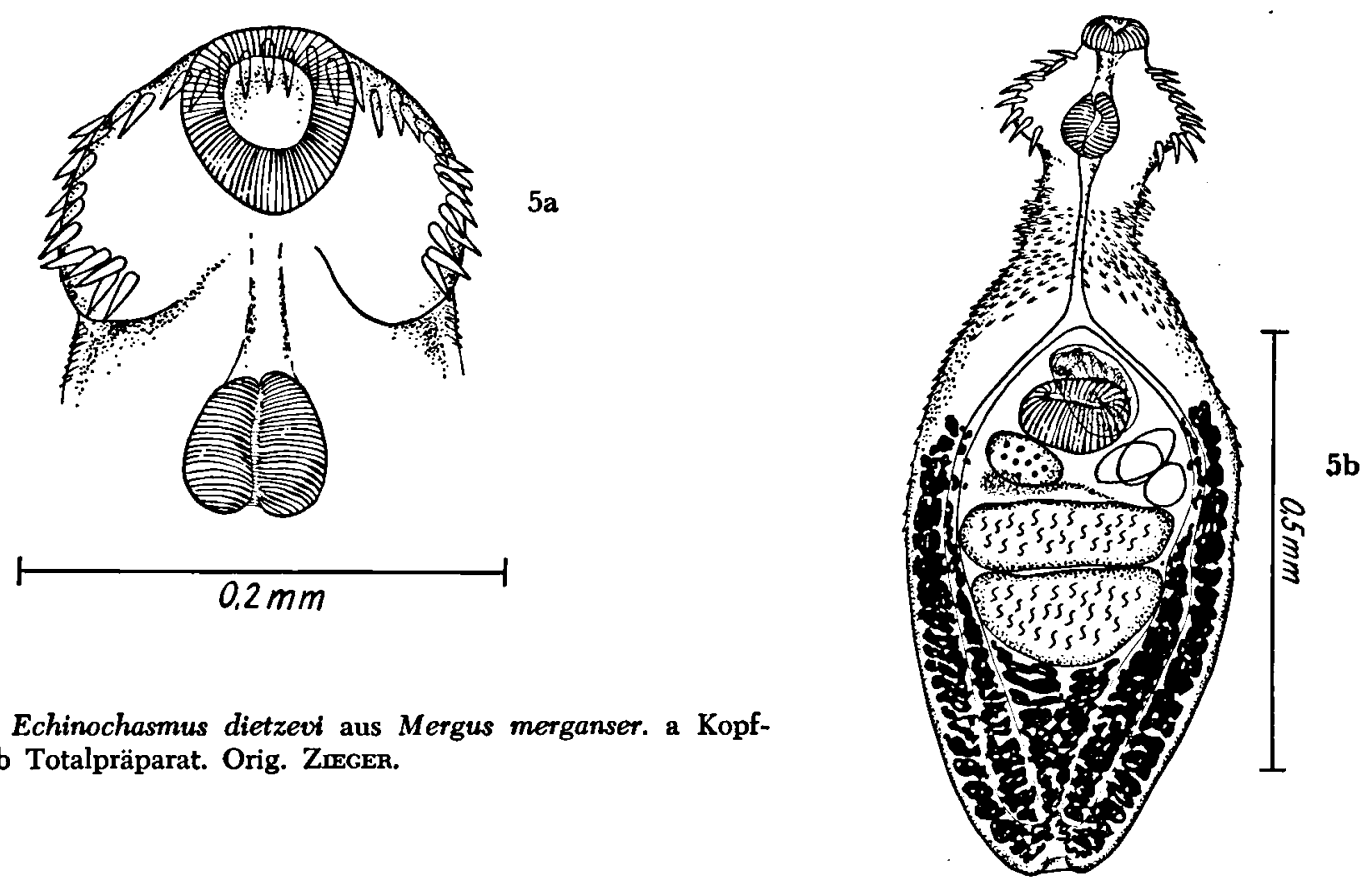

Abb. 5. Echinochasmus dietzevi aus Mergus merganser. a Kopfkragen, b Totalpräparat. Orig. ZIEGEr.

6. Echinochasmus mergi palaearcticus n. subsp.

Wirt/Herkunft: 1 ô Mergus $m$. merganser L. / aus der Umgebung von Berlin eingeliefert, Sektion am 3.4.1963 (nur 2 Gänsesäger untersucht).

Lokalisation: Vorderer und mittlerer Dünndarm.

Präparat-Nr.: kT 14/92-93 (31 Exemplare).

Beschreibung (vgl. Tabelle 4 und Abb. 6): Cuticula bestachelt; Körper mit nur sehr kurzem Halsteil, $0,99-2,015 \mathrm{~mm}$ lang bei einer maximalen Breite von 0,426-0,514 mm; Kopfkragen mit 22 Kragenstacheln; Praepharynx vorhanden, Pharynx sehr kräftig entwickelt, stets wesentlich länger und insgesamt größer als der Mundsaugnapf; Uterusbereich höchstens ein Drittel des Körperabschnitts hinter dem Bauchsaugnapf einnehmend; die Dotterstöcke reichen bis zum Vorderrand des vorderen Testis oder höchstens bis zur Höhe des Ovariums; Testes meist im mittleren Drittel des Hinterkörpers; Eigröße $90-$ $104 \times 55-62 \mu \mathrm{m}$.

Typus: Das der Abb. 6 zugrundeliegende Exemplar. Helminthensammlung Zoologische Forschungsstelle im Berliner Tierpark Nr. kT 14/92.

Bemerkungen. Die neue Subspecies unterscheidet sich von dem sehr ähnlichen nordamerikanischen Echinochasmus mergi (Cannon, 1939) n. comb., Syn. Stephanoprora mergi CANNON, $1939=$ Mesorchis mergi (Cannon) Sxrjabin \& Bašxurova, 1956 durch die größeren Maße von Saugnäpfen und Pharynx. Ahn-
Abb. 6. Echinochasmus mergi palaearcticus n. subsp. aus Mergus merganser, Typus. Orig. ZIEGER.
6

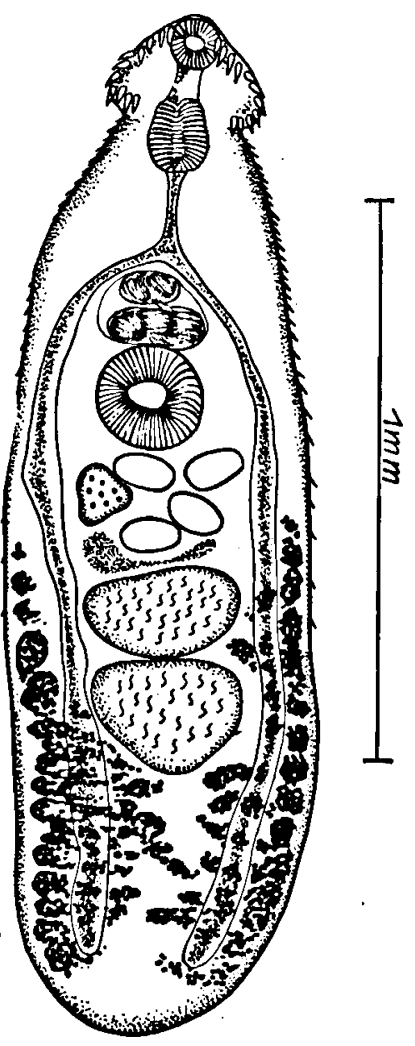


lich ist auch die nordamerikanische Ärt E. cochensi RAo, 1951, bei der aber - ebenso wie bei dem ebenfalls ähnlichen E. mirus MendHerm, 1940 - die Dotterstöcke weiter nach vorn reichen. Eine Zuteilung von E. mergi zur Gattung Mesorchis scheint mir nicht möglich, da die Dotterstöcke häufig die für Mesorchis angegebene vordere Grenze überschreiten und da die Körperform gedrungener ist und somit den charakteristischen Mesorchis-Arten nicht entspricht.

TABELle 4. Echinochasmus mergi palaearcticus n. subsp. aus Mergus merganser, Maße von 5 Exemplaren in $\mathrm{mm}$.

\begin{tabular}{|l|l|l|l|l|l|}
\hline $\begin{array}{l}\text { Körperlänge } \\
\text { max. Körperbreite }\end{array}$ & 2,015 & 1,261 & 1,422 & 1,466 & 1,230 \\
$\begin{array}{l}\text { Kopfkragen } \\
\text { Länge }\end{array}$ & 0,191 & 0,169 & 0,169 & 0,176 & 0,161 \\
$\begin{array}{l}\text { Breite } \\
\text { Mundsaugnapf } \\
\text { Länge }\end{array}$ & 0,272 & 0,220 & 0,257 & 0,242 & 0,220 \\
$\quad$ Breite & 0,066 & 0,072 & 0,076 & 0,069 & 0,069 \\
Bauchsaugnapf & 0,110 & 0,107 & 0,072 & 0,104 & 0,090 \\
$\quad$ Länge & 0,224 & 0,155 & 0,155 & 0,173 & 0,141 \\
Breite & 0,200 & 0,176 & 0,179 & 0,173 & 0,159 \\
$\begin{array}{l}\text { Pharynx } \\
\text { Länge }\end{array}$ & 0,104 & 0,110 & 0,121 & 0,117 & 0,097 \\
Breite & 0,110 & 0,079 & 0,076 & 0,086 & 0,069 \\
\hline
\end{tabular}

\section{Echinochasmus amphibolus KoTLÁN, 1922}

Synonyme: Echinochasmus botauri BAER, 1923; Echinochasmus bagulai Verma bei Macko (1960a).

Wirt/Herkunft: 2 ô Botaurus s. stellaris (L.) / aus der Umgebung von Berlin eingeliefert, Sektion am 11.12.1962 und 30.1.1963 (von Mitte 1959 bis 15.4. 19633 Große Rohrdommeln aus dem Gebiet untersucht).

Lokalisation: Vorderer und mittlerer Dünndarm.

Präparat-Nr.: kT 13/55-56 (11.12.62), kT 13/92-96 (30.1.63) (insgesamt 52 Exemplare).

Beschreibung (vgl. Tabelle 5 und Abb. 7): Cuticula bestachelt; Körper langgestreckt, mit sehr kurzem Halsteil, 1,048 - 2,566 $\mathrm{mm}$ lang bei einer maximalen Breite von 0,316 - 0,624 mm (meist auf der Höhe des Bauchsaugnapfs); Kopfkragen mit 24 Kragenstacheln; Bauchsaugnapf sehr groß, den Raum zwischen den Darmschenkeln fast völlig ausfüllend; Praepharynx bei größeren Exemplaren fallweise ziemlich lang; Uterusbereich ein Viertel bis ein Drittel des Hinterkörpers einnehmend; die Dotterstöcke rei- chen meist bis zur Höhe des Ovariums; Testes im mittleren Drittel oder im 2. Viertel des Hinterkörpers gelegen; Eigröße $72-97 \times 48-62 \mu \mathrm{m}$.

Bemerkungen. Die Art kommt in fischfressenden Vö-

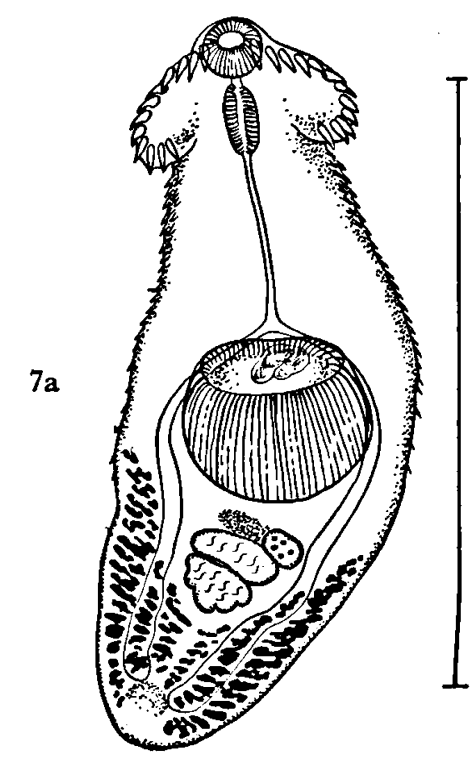

Abb. 7. Echinochasmus amphibolus aus Botaurus stellaris. $a$ und $b$ Praeadulti $c$, $d$ und $e$ verschiedener Entwicklungsstufe, e mit abnormer Kragenstachelzahl 25. Orig. Zieger.

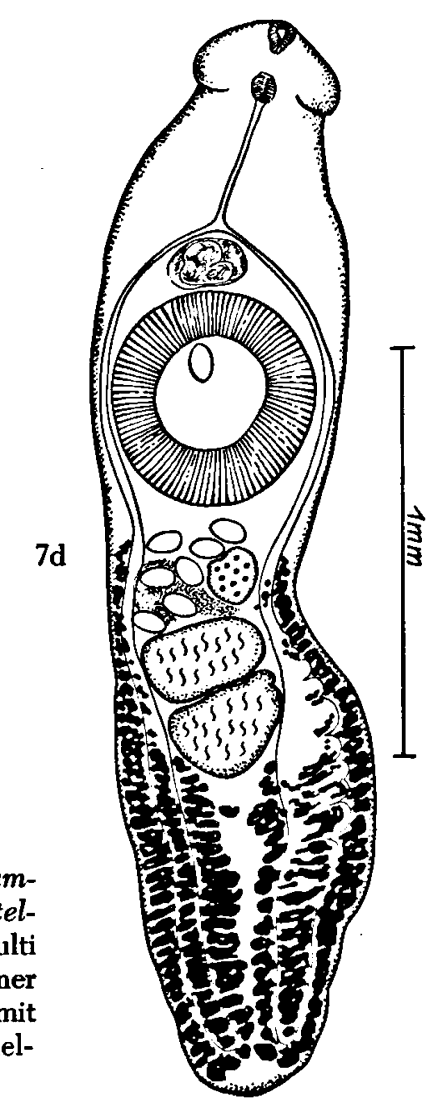


geln verschiedener Ordnungen vor (vgl. Macko 1960a, b, 1961/62, Sulgostowska 1960a und BychovskajaPavlovskaja 1962). Als Hilfswirte dürfen daher Fische, und zwar Süßwasserfische, vermutet werden.
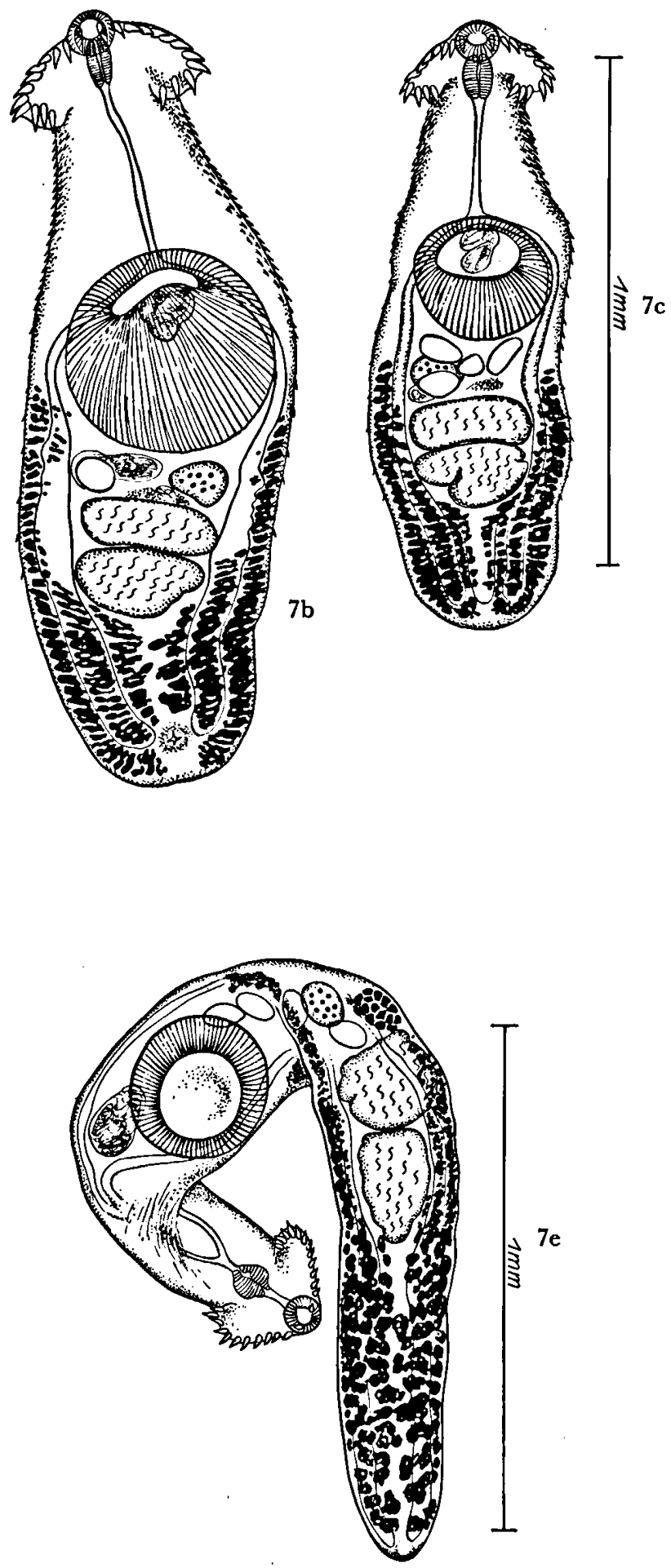

TABELLE 5. Echinochasmus amphibolus aus Botaurus stellaris, $\mathrm{Maße}$ von 5 Exemplaren in $\mathrm{mm}$.

\begin{tabular}{|l|l|l|l|l|l|}
\hline $\begin{array}{l}\text { Körperlänge } \\
\text { max. Körperbreite }\end{array}$ & 1,539 & 1,202 & 1,539 & 1,576 & 1,246 \\
$\begin{array}{l}\text { Kopfkragen } \\
\text { Länge }\end{array}$ & 0,396 & 0,404 & 0,528 & 0,433 & 0,411 \\
$\begin{array}{l}\text { Breite } \\
\text { Mundsaugnapf } \\
\text { Länge }\end{array}$ & 0,220 & 0,264 & 0,308 & 0,264 & 0,242 \\
$\begin{array}{l}\text { Breite } \\
\text { Bauchsaugnapf } \\
\text { Länge }\end{array}$ & 0,066 & 0,062 & 0,069 & 0,083 & 0,069 \\
$\begin{array}{l}\text { Breite } \\
\text { Pharynx }\end{array}$ & 0,066 & 0,083 & 0,093 & 0,083 & 0,083 \\
$\quad$ Länge & 0,276 & 0,259 & 0,293 & 0,304 & 0,276 \\
Breite & 0,252 & 0,276 & 0,311 & 0,290 & 0,273 \\
& 0,090 & 0,090 & 0,079 & 0,093 & 0,076 \\
& 0,059 & 0,055 & 0,110 & 0,059 & 0,062 \\
\hline
\end{tabular}

8. Echinochasmus beleocephalus (v. Linstow, 1873)

Wirt/Herkunft: Ardea c. cinerea L., Bucephala clangula (L.) / ein aus der Mark Brandenburg eingelieferter Graureiher, Sektion am 27.11.1962 (von Mitte 1959 bis 15.4.1963 wurden 8 Graureiher aus der Mark Brandenburg untersucht; als häufigste und typische Trematoden des Graureihers treten Apharyngostrigea cornu und Posthodiplostomum cuticola auf, siehe Odening 1962e und im Druck d); 1 q Schellente wurde frisch aus Hiddensee eingeliefert, Sektion am 15.1.1963 (nur 2 Schellenten untersucht, eine enthielt keine Helminthen).

Lokalisation: Dünndarm.

Präparat-Nr.: kT 13/61-62 (67 Exemplare aus Ardea), kT 14/27 (1 Exemplar aus Bucephala).

Beschreibung (vgl. Tabelle 6 und Abb. 8): Cuticula bestachelt; Körper mit nur kurzem Halsteil, 0,770 $1,283 \mathrm{~mm}$ lang, größte Breite 0,264 - 0,440 mm; Kopfkragen mit 24 Kragenstacheln; Dotterstöcke bis zur Höhe des Bauchsaugnapfs reichend; unmittelbar hinter dem Bauchsaugnapf besteht die Tendenz zur Vereinigung der Dotterstöcke beider Körperseiten; Testes im mittleren Drittel oder 2. Viertel des Hinterkörpers; Eigröße $69-83 \times 45-52 \mu \mathrm{m}$.

Bemerkungen. Die Art ist aus fischfressenden Vögeln verschiedener Ordnungen (Ardeiformes, Anseriformes) sowie aus Hausente und Haushuhn (Fütterung mit Fischresten!) bekannt (vgl. BychovsKaja-PavlovsKaja 1962, Bezubik 1956a, Macko 1960b, 1961/62, SulcostowsKa 1960a und Reimer 1962). 
K. ODENING

TABELlE 6. Echinochasmus beleocephalus. Maße von 6 Exemplaren in mm.

\begin{tabular}{|c|c|c|c|c|c|c|}
\hline Wirt & Bucephala & Mergus & & & & \\
\hline Körperlänge & 0,821 & 0,770 & 0,843 & 0,770 & 0,975 & 0,843 \\
\hline max. Körperbreite & 0,308 & 0,440 & 0,360 & 0,264 & 0,323 & 0,330 \\
\hline \multicolumn{7}{|l|}{ Kopfkragen } \\
\hline Länge & 0,103 & 0,110 & 0,139 & 0,110 & 0,132 & 0,132 \\
\hline Breite & 0,154 & 0,264 & 0,206 & 0,169 & 0,220 & 0,213 \\
\hline \multicolumn{7}{|l|}{ Mundsaugnapf } \\
\hline Länge & 0,066 & 0,062 & 0,062 & 0,055 & 0,059 & 0,062 \\
\hline Breite & 0,055 & 0,079 & 0,072 & 0,052 & 0,038 & 0,069 \\
\hline \multicolumn{7}{|l|}{ Bauchsaugnapf } \\
\hline Länge & 0,155 & 0,131 & 0,124 & 0,114 & 0,128 & 0,124 \\
\hline Breite & 0,138 & 0,166 & 0,138 & 0,121 & 0,117 & 0,135 \\
\hline \multicolumn{7}{|l|}{ Pharynx } \\
\hline Länge & 0,072 & 0,079 & 0,072 & 0,055 & 0,069 & 0,069 \\
\hline Breite & 0,072 & 0,066 & 0,062 & 0,041 & 0,041 & 0,062 \\
\hline
\end{tabular}
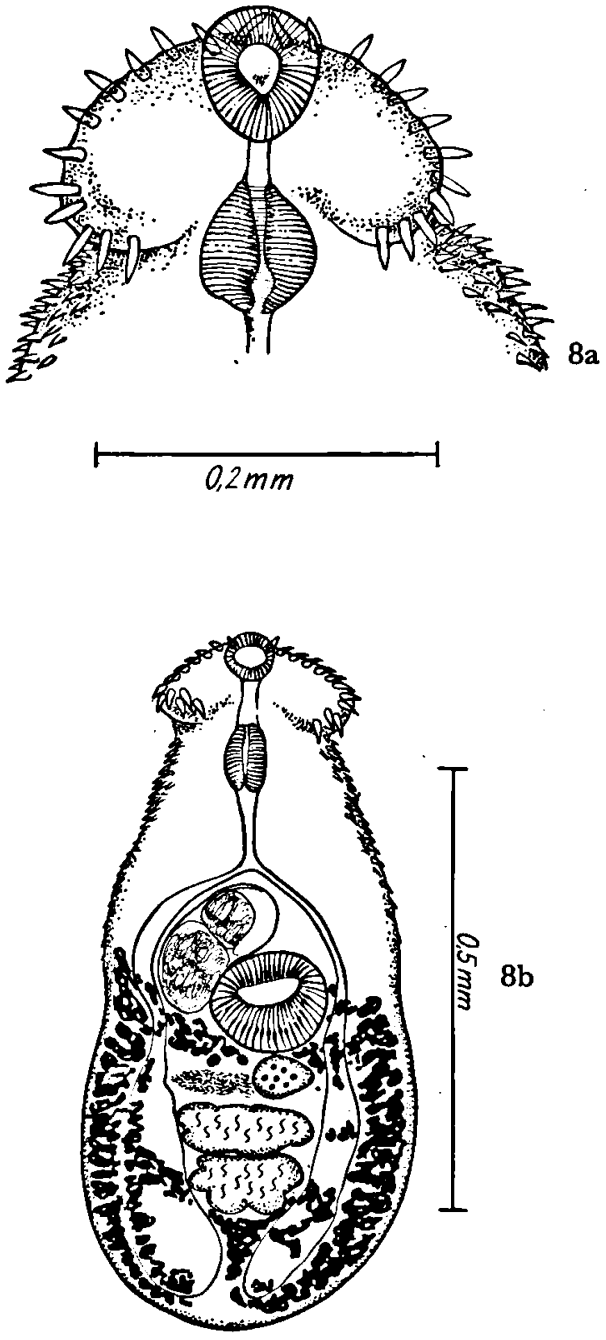

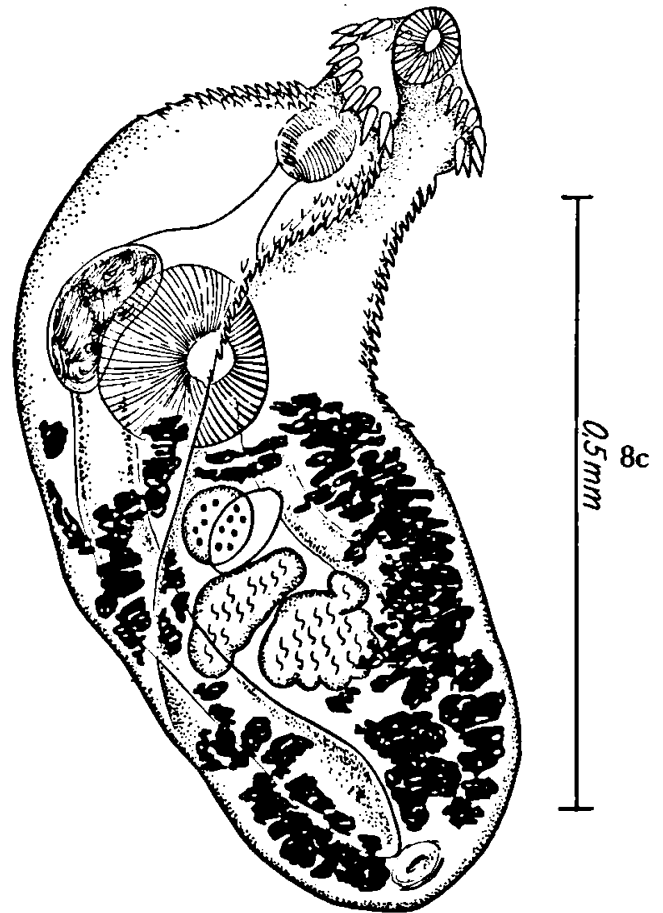

Abb. 8. Echinochasmus beleocephalus. a Kopfkragen eines Exemplars aus Ardea cinerea, b aus Ardea cinerea, c aus Bucephala clangula. Orig. ZreGER. 


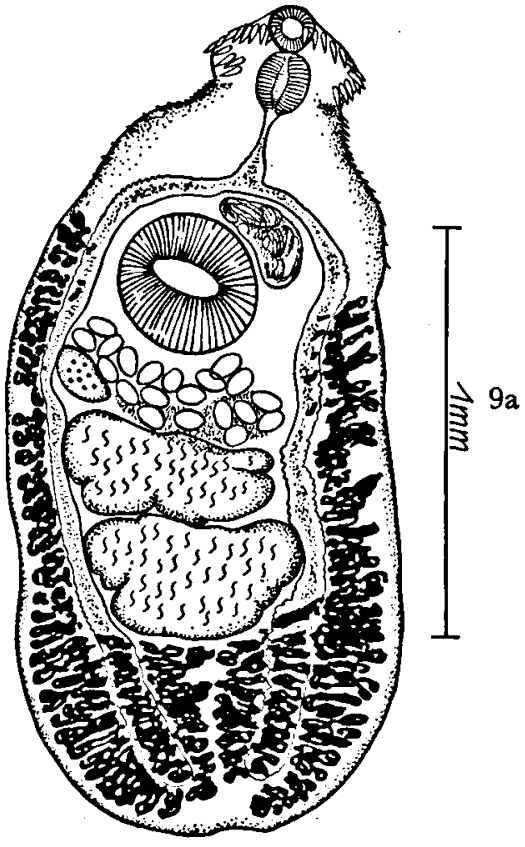

9. Echinochasmus coaxatus DIETz, 1909

Synonym: Echinochasmus squamatus MENDHEIM, 1940.

Wirt/Herkunft: 2 Podiceps c. cristatus (L.) / BerlinRummelsburg und Umgebung von Berlin, Sektion am 24.5.1962 und 25.2.1963.

Lokalisation: Dünndarm.

Präparat-Nr.: kT 11/39, 41-42 (20 Exemplare vom 24.5.62), kT 14/67-68 (13 Exemplare vom 25.2.63). Beschreibung (vgl. Tabelle 7 und Abb. 9): Cuticula bestachelt; Körper meist gedrungen erscheinend, mit kurzem, aber stark verschmälertem Halsteil, 1,209 $2,785 \mathrm{~mm}$ lang bei einer maximalen Breite von 0,661 - 1,026 mm (auf der Höhe der Testes); Kopfkragen mit 24 Kragenstacheln; die Dotterstöcke reichen bis zur Höhe des Bauchsaugnapfs; Uterusbereich kurz, höchstens ein Viertel bis ein Fünftel des Hinterkörpers einnehmend; Testes in der vorderen Hälfte oder im mittleren Drittel des Hinterkörpers; Eigröße $76-93 \times 45-59 \mu \mathrm{m}$.

Bemerkungen. Auf Grund des mir vorliegenden Materials ist die Selbständigkeit von $E$. squamatus nicht

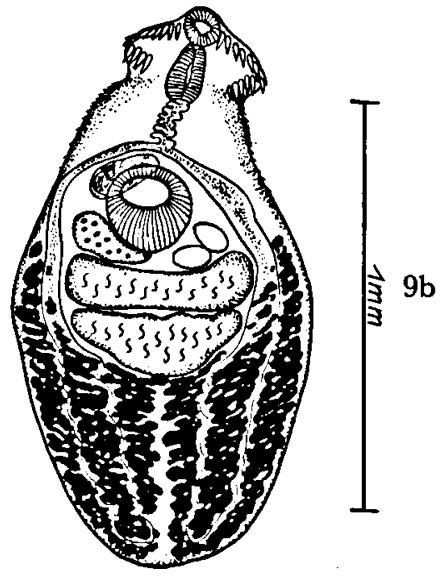

Abb. 9. Echinochasmus coaxatus aus Podiceps cristatus. Exemplars aus Ardea cinerea, b aus Ardea cinerea, c aus Bucephala clangula. Orig. ZrEger.

mehr aufrechtzuerhalten (vgl. ODENIng im Druck a). $\mathrm{Zu}$ weiteren mitteleuropäischen Funden der Art siehe Reimer (1962), Macko (1959, 1961/62) und SulgoSTowsKa (1958, 1960a). MACKo (1959) bildete bereits einen Kopfkragen mit der abweichenden Kragenstachelzahl $25 \mathrm{ab}$; diese Anomalie scheint öfters vorzukommen (vgl. Abb. 9 b), sie hat jedoch keinen Einfluß auf die dorsale Unterbrechung der Kragensta. chelreihe.

TABelle 7. Echinochasmus coaxatus aus Podiceps cristatus, Maße von 5 Exemplaren in $\mathrm{mm}$.

\begin{tabular}{|l|l|l|l|l|l|}
\hline $\begin{array}{l}\text { Körperlänge } \\
\text { max. Körperbreite }\end{array}$ & 2,116 & 1,598 & 2,639 & 2,639 & 2,697 \\
$\begin{array}{l}\text { Kopfkragen } \\
\text { Länge }\end{array}$ & 0,916 & 0,953 & 1,026 & 0,938 \\
$\begin{array}{l}\text { Breite } \\
\text { Mundsaugnapf } \\
\text { Länge }\end{array}$ & 0,228 & 0,161 & 0,206 & 0,220 & 0,220 \\
$\begin{array}{l}\text { Breite } \\
\text { Bauchsaugnapf } \\
\text { Länge }\end{array}$ & 0,128 & 0,121 & 0,124 & 0,138 & 0,138 \\
$\begin{array}{l}\text { Breite } \\
\text { Pharynx }\end{array}$ & 0,131 & 0,104 & 0,152 & 0,145 & 0,141 \\
$\quad$ Länge & 0,335 & 0,293 & 0,345 & 0,335 & 0,345 \\
Breite & 0,351 & 0,345 & 0,361 & 0,351 & 0,358 \\
\hline
\end{tabular}



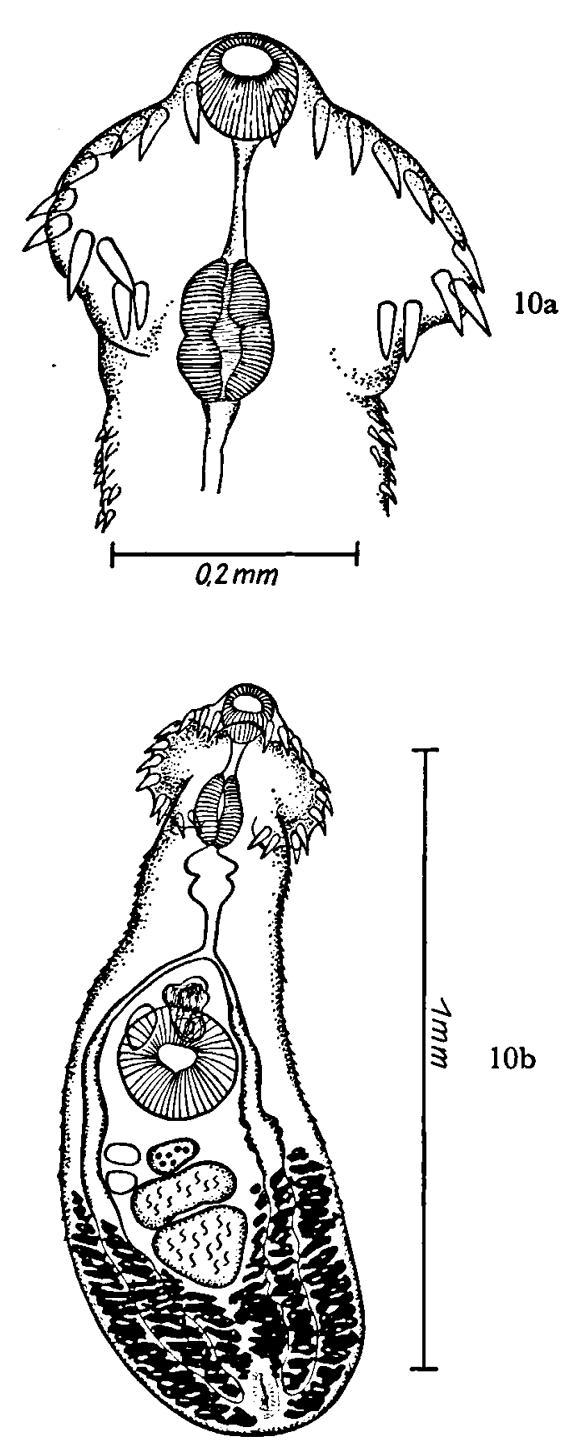

10. Monilifer spinulosus (RUDOLPHI, 1809)

Synonyme: Echinostomum spinosum ODHNER, 1910; Stephanoprora spinosa (ODHNER) bei MENDHEIM (1940;) Echinochasmus (Echinochasmus) spinosus (OdHNER) bei Skrjabin \& BaŠxivora (1956).

Wirte/Herkunft: Gavia arctica (L.), Podiceps c. cristatus (L.), Podiceps r. ruficollis (Pallas)/ 19 und $1 \hat{\sigma}$ Prachttaucher wurden aus der DDR eingeliefert, Sektion am 1. und 6.12.1962 (bis 15.4.1963 insgesamt 3 Prachttaucher untersucht); 6 Fälle bei Haubentauchern und ein Fall beim Zwergtaucher (die Lappentaucher stammten sämtlich aus Berlin und Umgebung).

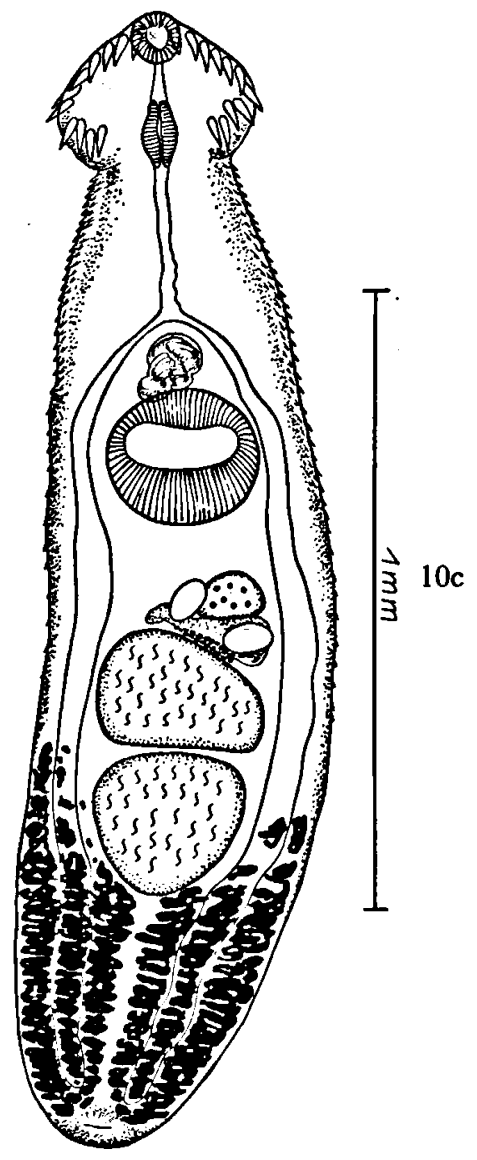

Abb. 10. Monilifer spinulosus aus Gavia arctica. a Kopfkragen, b "spinulosus"-Form, c "spinosus"-Form. Orig. ZIEGER.

Lokalisation: Gesamter Darm von Pylorus bis Kloake (Gavia), Dünndarm (Podiceps).

Präparat-Nr.: kT 13/46, 58-59 (insgesamt 27 Exemplare aus Gavia), kT 3/78-79, kT 11/43, kT 13/89, kT 14/2, kT 14/6, kT 14/70, $72-73$ (insgesamt 141 Exemplare aus Podiceps cristatus), kT 9/77-81 (15 Exemplare aus Podiceps ruficollis).

Beschreibung (vgl. Tabelle 8 und Abb. 10): Cuticula vor allem im Vorderkörper kräftig bestachelt; Körperlänge bis $2 \mathrm{~mm}$, maximale Körperbreite bis $0,7 \mathrm{~mm}$; Körperform sehr variabel, im Umriß dick keulen- oder flaschenförmig bis gleichmäßig oblong oder mehr oder weniger lanzettförmig; vorderer Testis stets mehr oder weniger breitgestreckt, hinterer Testis 
stets dreieckig oder herzförmig mit nach hinten gerichteter Spitze, von den Dotterstöcken seitlich eingefaßt; Uterusbereich meist ziemlich kurz; Eigröße $59-90 \times 37-59 \mu \mathrm{m}$.

Bemerkungen. Die Art ist in Mitteleuropa bei Podicipedidae häufig (siehe BEzuBIK 1956a, SulgostowsKa 1958, 1960a, Macxo 1959, 1961/62, Odening 1962 c, e und im Druck a). Nach MENDhEIM (1940) soll sie gelegentlich auch in verschiedenen Möwenarten vorkommen (leider ohne nähere Angaben). Demnach kommt M. spinulosus in Podicipediformes, Gaviiformes und Lari (?) vor, als Hilfswirte sind Fische anzunehmen. Die Körperform der Art ist sehr veränderlich. Sie variiert von der von Dietz (1910) für $M$. spinulosus (RuDoLPHI) angegebenen Form mit rundlichem Hinterende bis zu der von OdHNER (1910) für "Echinostomum spinosum ODHNER ${ }^{\text {"E dargestellten }}$ länglichen Form, und darüber hinaus bis zu noch schlankeren Formen. Bei diesen letztgenannten Formen ist eine Trennung von Mesorchis denticulatus (RudolpH, 1802) schwierig, hier hilft nur die Form der Testes. Skrjabin \& BaŠKirova (1956) stellten spinosus ODHNER und spinulosus RuDOLPHI in zwei verschiedene Untergattungen von Echinochasmus! Dieses Verfahren steht in zweifacher Weise im Widerspruch zu den von SKRJABIN \& BAŠKIROva selbst vertretenen Einteilungsprinzipien für die Echinochasminae-Gattungen nach der vorderen Ausdehnung der Dotterstöcke: 1. Monilifer zeigt die gleiche Ausdehnung der Dotterstöcke wie Mesorchis; es ist daher unverständlich, weshalb Monilifer als Untergattung von Echinochasmus aufgefaßt wurde. 2. Die ODHNersche Art spinosus entspricht in der Ausdehnung der Dotterstöcke nicht der Diagnose der Untergattung Echinochasmus sensu SkrJabin \& BAŠKIROva. Es gibt meines Erachtens nur die folgenden Möglichkeiten für die Einordnung von Monilifer bzw. der einzigen dieser Gattung zugrundeliegenden Art M. spinulosus =spinosus: entweder eigene Gattung, die in ihren Merkmalen zwischen Echinochasmus (Körperform) und Mesorchis (Ausdehnung der Dotterstöcke) steht, oder Untergattung von Mesorchis, oder Synonym von Mesorchis (Auffassung von MENDHEIM 1940, 1943). Ich ziehe gegenwärtig die erstgenannte Lösung vor.

TABelle 8. Monilifer spinulosus. Maße von 10 Exemplaren in mm.

\begin{tabular}{|c|c|c|c|c|c|c|c|c|c|c|}
\hline Körperform & \multicolumn{5}{|c|}{ schlank, länglich (,,spinosus“) } & \multicolumn{5}{|c|}{ gedrungen, keulenförmig (,,spinulosus“) } \\
\hline Wirt & \multirow{3}{*}{$\frac{\mid \text { Podiceps }}{\mid \begin{array}{c}2,228 \\
0,492\end{array}}$} & \multicolumn{4}{|c|}{ Gavia } & \multirow{2}{*}{$\frac{\mid \text { Podiceps }}{1,151}$} & \multicolumn{4}{|c|}{ Gavia } \\
\hline Körperlänge & & 1,906 & 1,869 & 1,795 & 1,906 & & 1,369 & 1,209 & 1,422 & 1,319 \\
\hline $\begin{array}{l}\text { max. Körperbreite } \\
\text { Kopfkragen }\end{array}$ & & 0,404 & 0,462 & 0,469 & 0,382 & 0,426 & 0,499 & 0,440 & 0,316 & 0,470 \\
\hline Länge & 0,235 & 0,257 & 0,242 & 0,257 & 0,250 & 0,147 & 0,206 & 0,257 & 0,198 & 0,198 \\
\hline Breite & 0,308 & 0,345 & 0,330 & 0,338 & 0,330 & 0,272 & 0,286 & 0,294 & 0,286 & 0,272 \\
\hline Mundsaugnapf & & & & & & & & & & \\
\hline Länge & 0,086 & 0,121 & 0,100 & 0,114 & 0,117 & 0,076 & 0,086 & 0,097 & 0,079 & 0,076 \\
\hline Breite & 0,097 & 0,093 & 0,110 & 0,104 & 0,093 & 0,104 & 0,104 & 0,086 & 0,090 & 0,104 \\
\hline Bauchsaugnapf & & & & & & & & & & \\
\hline Länge & 0,231 & 0,266 & 0,231 & 0,235 & 0,242 & 0,207 & 0,190 & 0,190 & 0,190 & 0,193 \\
\hline Breite & 0,221 & 0,266 & 0,255 & 0,245 & 0,248 & 0,248 & 0,242 & 0,190 & 0,207 & 0,207 \\
\hline Pharynx & & & & & & & & & & \\
\hline Länge & 0,104 & 0,117 & 0,114 & 0,104 & 0,110 & 0,104 & 0,100 & 0,114 & 0,076 & 0,104 \\
\hline Breite & 0,110 & 0,079 & 0,069 & 0,069 & 0,072 & 0,076 & 0,079 & 0,072 & 0,097 & 0,076 \\
\hline Vorderer Testis & & & & & & & & & & \\
\hline Länge & 0,190 & 0,173 & 0,193 & 0,148 & 0,173 & 0,097 & 0,104 & 0,076 & 0,124 & 0,104 \\
\hline Breite & 0,361 & 0,224 & 0,266 & 0,248 & 0,242 & 0,200 & 0,255 & 0,179 & 0,155 & 0,242 \\
\hline Hinterer Testis & & & & & & & & & & \\
\hline Länge & 0,262 & 0,224 & 0,242 & 0,190 & 0,214 & 0,135 & 0,148 & 0,131 & 0,155 & 0,128 \\
\hline Breite & 0,321 & 0,207 & 0,242 & 0,207 & 0,204 & 0,148 & 0,228 & 0,155 & 0,162 & 0,138 \\
\hline
\end{tabular}




\section{Mesorchis pseudoechinatus (OLsson, 1876)}

Wirt/Herkunft: 1 Podiceps c. cristatus (L.), 1 Gavia arctica (L.) / aus der Umgebung von Berlin bzw. der DDR eingeliefert, Sektion am 25.2.1963 (Podiceps) und 6.12.1962 (Gavia).

Lokalisation: Dünndarm.

Präparat-Nr.: kT 14/63 (1 Exemplar aus Podiceps), kT 13/60 (1 adultes und mehrere präadulte Exemplare aus Gavia).

Beschreibung (vgl. Abb. 11; Maßangaben für das adulte Exemplar aus Gavia in Klammern): Cuticula der vorderen Körperhälfte bestachelt; Körper langgestreckt, $5,864(3,372) \mathrm{mm}$ lang bei einer maximalen Breite von $0,697(0,624) \mathrm{mm}$; Kopfkragen 0,382 $(0,330) \mathrm{mm}$ lang und $0,477(0,440) \mathrm{mm}$ breit, mit 22 Kragenstacheln; Mundsaugnapf 0,206 $\times 0,191$ $(0,145 \times 0,124) \mathrm{mm}$ groß; Bauchsaugnapf $0,433 \times$ $0,440(0,311 \times 0,371) \mathrm{mm}$ groß; Pharynx $0,184 \times$ $0,147(0,124 \times 0,104) \mathrm{mm}$ groß; Dotterstöcke bis zur Höhe des vorderen Testis reichend; Testes läng-

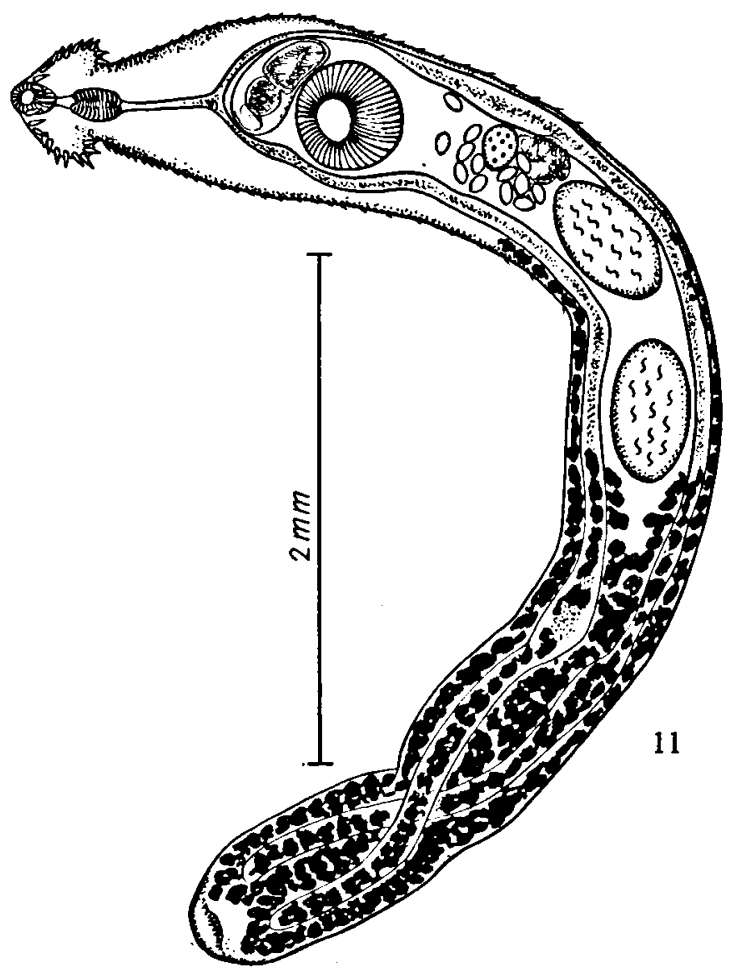

lich-elliptisch, vor der Körpermitte gelegen; vorderer Testis $0,543(0,328) \mathrm{mm}$ lang und $0,308(0,276) \mathrm{mm}$ breit, hinterer Testis $0,551(0,328) \mathrm{mm}$ lang und $0,294(0,269) \mathrm{mm}$ breit; Eigröße 86 - $100 \times 59-$ $62 \mu \mathrm{m}$.

Bemerkungen. Die Art war ursprünglich aus Lari beschrieben worden, wurde später aber auch aus Podicipedidae (vgl. Macko 1959, 1961/62 und SuLgostowsKa 1958, 1960a) und aus Anseriformes (vgl. Bychovskaja-Pavlovskaja 1962) bekannt. Reimer (1962) stellte die Art auf Hiddensee bei Vögeln aller 3 Ordnungen fest, dazu noch in Gavia stellata. Als Hilfswirte dienen Fische.

\section{Episthmium colymbi ŠIGIN, 1956?}

Synonyme: Echinochasmus (Episthmium) mathevossianae S̆ACHTACHTINSXaja in KURAŠviLI, 1957; Echinochasmus (Episthmium) schigini ByChOvSKaja-PavLOVSKAJA, 1962 nom. n. pro Echinochasmus (Episthmium) colymbi SIGIN, 1956.

Wirt/Herkunft: Podiceps c. cristatus (L.), 1 / aus der Umgebung von Berlin eingeliefert, Sektion am 25.2.1963 (von Mitte 1959 bis 15.4.1963 wurden 10 Haubentaucher aus dem Gebiet von Berlin untersucht).

Lokalisation: Dünndarm.

Präparat-Nr.: kT 14/67, 69 (3 Exemplare).

Beschreibung (vgl. Tabelle 9 und Abb. 12): Cuticula kräftig bestachelt; Körper länglich-oval oder länglichelliptisch, mit leicht konischem Hinterende; Kopfkragen nur wenig hervortretend, mit 22 (?) Kragenstacheln (Zählung nach 2 lebenden Exemplaren; bei beiden Exemplaren ist im Präparat der Kragen beschädigt, beim 3. Exemplar fehlt der Vorderkörper); ein besonders abgesetztes Halsteil fehlt; Dotterstöcke vor dem Bauchsaugnapf in einer charakteristischen Pyramide sich vereinigend; Uterusbereich etwa das 1. Drittel des Hinterkörpers einnehmend; Testes breitgestreckt, im mittleren Drittel des Hinterkörpers gelegen; Eigröße $83-100 \times 45-55 \mu \mathrm{m}$.

Bemerkungen. Es ist anzunehmen, da $B$ ursprünglich 24 Kragenstacheln vorhanden waren. Zur Art vgl. Macko (1959, 1961/62) und SulgostowsKa (1960a). Sie wurde bisher in Podicipedidae und Anatidae gefunden.
Abb. 11. Mesorchis pseudoechinatus aus Podiceps crista tus. Orig. ZIEGER. 


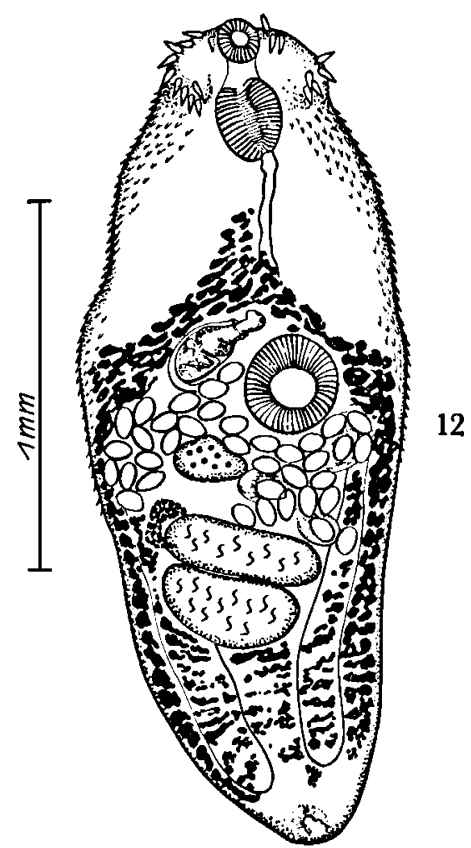

Abb. 12. Episthmium colymbi aus Podiceps cristatus. Orig. Zneger.

TABelle 9. Episthmium colymbi aus Podiceps cristatus, Maße der 3 Exemplare in $\mathrm{mm}$.

\begin{tabular}{|l|c|c|c|}
\hline Körperlänge & 2,529 & 2,214 & - \\
max. Körperbreite & 1,026 & 0,865 & 0,916 \\
Kopfkragen & & & \\
Länge & 0,308 & 0,257 & - \\
Breite & 0,675 & 0,477 & - \\
$\begin{array}{l}\text { Mundsaugnapf } \\
\text { Länge }\end{array}$ & 0,090 & 0,121 & - \\
Breite & 0,155 & 0,148 & - \\
Bauchsaugnapf & 0,276 & 0,276 & 0,276 \\
Länge & 0,311 & 0,252 & 0,259 \\
$\begin{array}{l}\text { Breite } \\
\text { Pharynx } \\
\text { Länge }\end{array}$ & 0,221 & 0,179 & - \\
Breite & 0,214 & 0,155 & - \\
Vorderer Testis & 0,214 & 0,141 & 0,210 \\
Länge & 0,499 & 0,447 & 0,385 \\
Breite & 0,259 & 0,197 & 0,276 \\
Hinterer Testis & 0,423 & 0,372 & 0,392 \\
Länge & & & \\
Breite & & & \\
\hline
\end{tabular}

Microparyphiinae MENDHEIM, 1943

13. Ignavia renalis orientalis $\mathrm{n}$. subsp.

Wirt/Herkunft: Pelecanus philippensis GMELIN / im- portiert aus der Indischen Union am 31.7.1961, Sektion am 9.4.1963.

Lokalisation: Ureteren.

Präparat-Nr.: kT 14/95-98 (4 Exemplare in Bruchstücken).

Beschreibung (vgl. Tabelle 10 und Abb. 13): Cuticula bis zum Bereich des hinteren Testis bestachelt; Körper langgestreckt mit zugespitztem Hinter- und Vorderende, 15,7 - 16,8 mm lang bei einer maximalen Breite im Bereich hinter dem hinteren Testis von 2,4-3 mm; vor dem Mundsaugnapf befindet sich ein papillenförmiger Fortsatz; Kopfkragen breitgestreckt elliptisch mit jederseits 10 Kragenstacheln, deren einreihige Anordnung durch einen breiten dorsalen Zwischenraum in der Mitte unterbrochen wird; Kragenstacheln $35-65 \mu \mathrm{m}$ lang, maximale Breite an der Basis $16-26 \mu \mathrm{m}$; Ovarium breitgestreckt oval, elliptisch oder aus 3 großen Lappen bestehend, median oder submedian gelegen; Testes rundlich oder queroval, mit ziemlich tiefen Einkerbungen; Eigröße $79-95 \times 44-59 \mu \mathrm{m}$.

Bemerkungen. Die vorliegende Art unterscheidet sich von Ignavia aquilae (OŠmarin \& Belous, 1951) und I. ardeae (ŠEvČENKO, 1954) durch wesentlich größere Körper- und Organmaße. Von diesen beiden aus der UdSSR beschriebenen Arten und von der amerikanisch-afikanischen 1 . venusta (TeIxeIRA de FreItas, 1948), der typischen Art des Genus, unterscheidet sich die vorliegende indische Form durch einen relativ kürzeren Ösophagus und größeren Pharynx. Überraschenderweise stimmen Habitus, Körper- und Organmaße und andere Einzelheiten (z.B. der papillenförmige Fortsatz am Körpervorderende) der indischen Form mit I. renalis (YEH, 1954) überein. I. renalis war beschrieben worden aus einem nordamerikanischen Pelecanus erythrorhynchos, der im Londoner Zoo gestorben war. Allerdings sind die Kragenstacheln der indischen Form kleiner, weshalb ich diese als Unterart von $I$. renalis auffasse: Ignavia renalis orientalis $\mathbf{n}$. subsp.

Typus: Das der Abb. 13 b zugrundeliegende Exemplar, Helminthensammlung Zoologische Forschungsstelle im Berliner Tierpark Nr. kT 14/95.

Uberblick über das Genus Ignavia TEIXEIRA DE FREITAS, 1948

Ursprünglich aufgestellt mit den Arten Ignavia venusta (typische Art) und 1 . inops (Freitas, 1948). Letztgenannte Art gehört aber nicht hierher und wurde dementsprechend von YaMaGuTI (1958) nicht mit in die Gattung einbezogen. Yamaguti (1958) betrachtet Nephroechinostoma OŠmarin \& BeLous, 1951 
K. ODENING

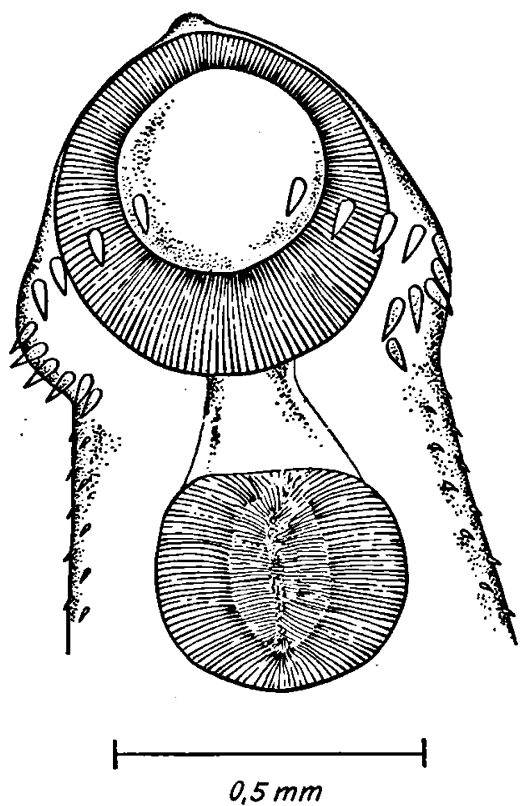

als Synonym von Ignavia, was völlig verständlich ist. Sowohl OŠmarin \& Belous und SKRJABIN \& BaŠKIROVA (1956) als auch YAMAGUTI (1958) betrachten Nephroechinostoma bzw. Ignavia als einziges Genus einer eigenen Unterfamilie (Nephroechinostomatinae Ošmariv \& Belous, 1951 = Ignaviinae YAmagutr, 1958). Ignavia renalis wurde ursprünglich als Allechinostomum renale YEH, 1954 beschrieben. Bei SKRJABIN und BAŠKIRova (1956) sind die Vertreter der Gattung Ignavia auf 3 verschiedene Gattungen in 3 verschiedenen Unterfamilien verteilt. Nach der Ausbildung des Kopfkragens teile ich Ignavia der bisher nur eine Gattung umfassenden Unterfamilie Microparyphiinae Mendheim, $1943 \mathrm{zu}$. Die Gattung stellt eine morphologisch und biologisch (Nierenparasiten!) gut umrissene natürliche Gruppe dar. Es sind folgende Arten bekannt:

Ignavia venusta TEIXEIRA DE FreItAS, 1948 (Südamerika; Nordamerika, siehe LuMSDEN \& Zuscike 1963; Afrika, siehe Wrigrr 1957);

I. aquilae (OŠmarnN \& Belous, 1951), Syn. Nephroechinostoma aquilae OŠMARIN \& BELOUS, 1951 (UdSSR);

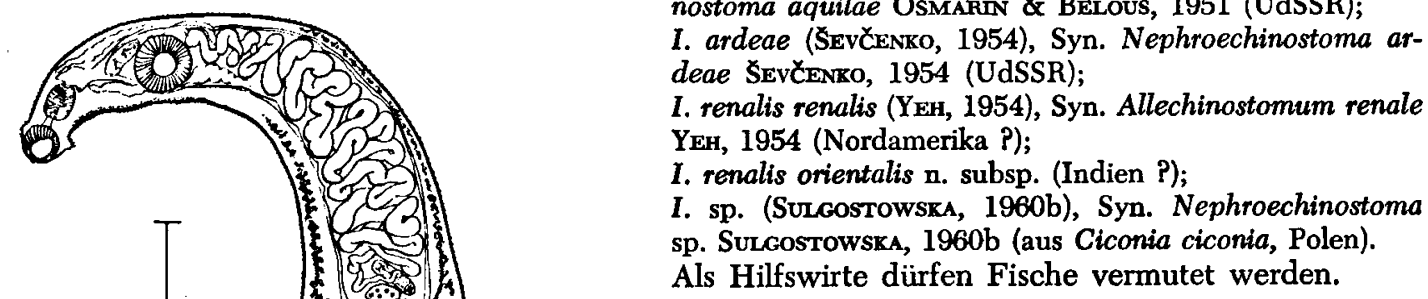

TABelle 10. Ignavia renalis orientalis n.subsp. aus Pelecanus philippensis, Maße von 3 zusammengesetzten Exemplaren in $\mathrm{mm}$.

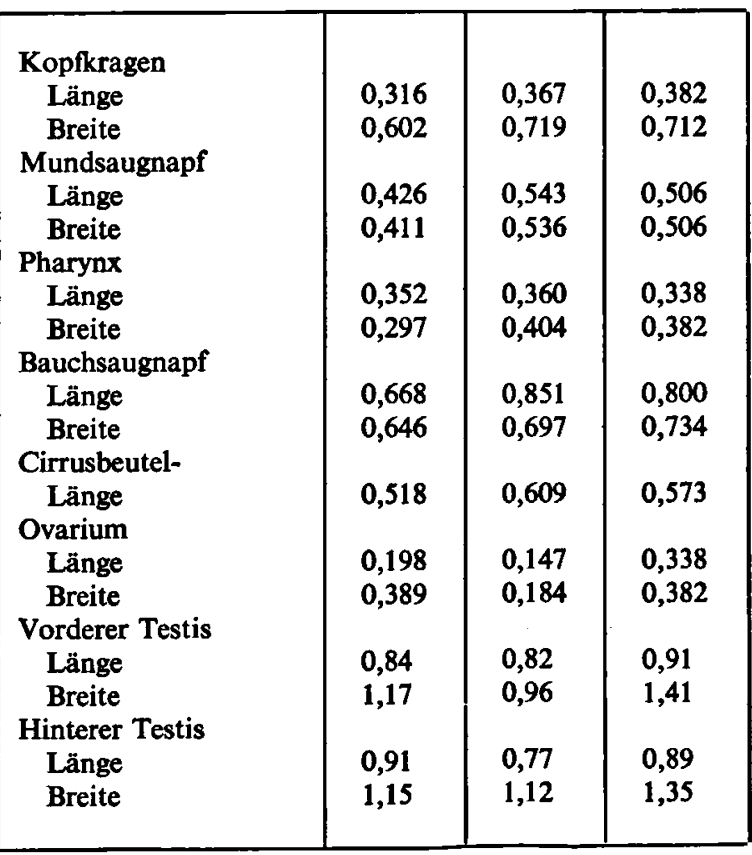

Abb. 13. Ignavia renalis orientalis n. subsp., aus Pelecanus philippensis. a Kopfkragen (man beachte den papillenförmigen Fortsatz am Körpervorderende), b Typus. Orig. ZIEGER. 
Nach Drucklegung dieser Arbeit erfuhr ich von einer Arbeit (Gupta 1962; Ref. Ž. 196316 K 24), in der eine neue indische (?) Art Ignavia breviovari(c)a aus Ardea purpurea (L.) beschrieben wurde.

Psilostomatidae OdHNER, 1913

\section{Psilostomum brevicolle (CREPLIN, 1829)}

Synonym: Distomum platyurum MüHLING, 1892.

Wirt/Herkunft: 1 q Somateria mollisima (L.) / eingeliefert von der Insel Hiddensee, Sektion am 6.2. 1963.

Lokalisation: Dünndarm.

Präparat-Nr.: kT 15/26 (1 Exemplar).

Beschreibung vgl. Abb. 14): Körper länglich-oval, $1,724 \mathrm{~mm}$ lang, maximale Breite $0,543 \mathrm{~mm}$; Mundsaugnapf $0,242 \times 0,235 \mathrm{~mm}$ groß; Pharynx $0,159 \mathrm{~mm}$ im Durchmesser; Ösophaguslänge 0,069 mm; Bauchsaugnapf $0,200 \times 0,224 \mathrm{~mm}$ groß; Ovarium rundlichoval, glattrandig, $0,086 \mathrm{~mm}$ lang und $0,1 \mathrm{~mm}$ breit; Testes im hinteren Körperdrittel, glattrandig, breitgestreckt; vorderer Testis $0,190 \mathrm{~mm}$ lang und 0,293 $\mathrm{mm}$ breit, hinterer Testis $0,231 \mathrm{~mm}$ lang und 0,276 mm breit.

Bemerkungen. Bei dieser Art soll ein Osophagus fehlen (siehe jedoch Abb. 14!). Der Entwicklungszyklus der Art wurde von Reimer (1962) auf Hiddensee vollständig erforscht. Die Entwicklung verläuft im Brackwasser der Ostsee. Reimer stellte auf Hiddensee natürlichen Befall bei Clangula hyemalis, Melanitta fusca, M. nigra und Aythya marila fest.

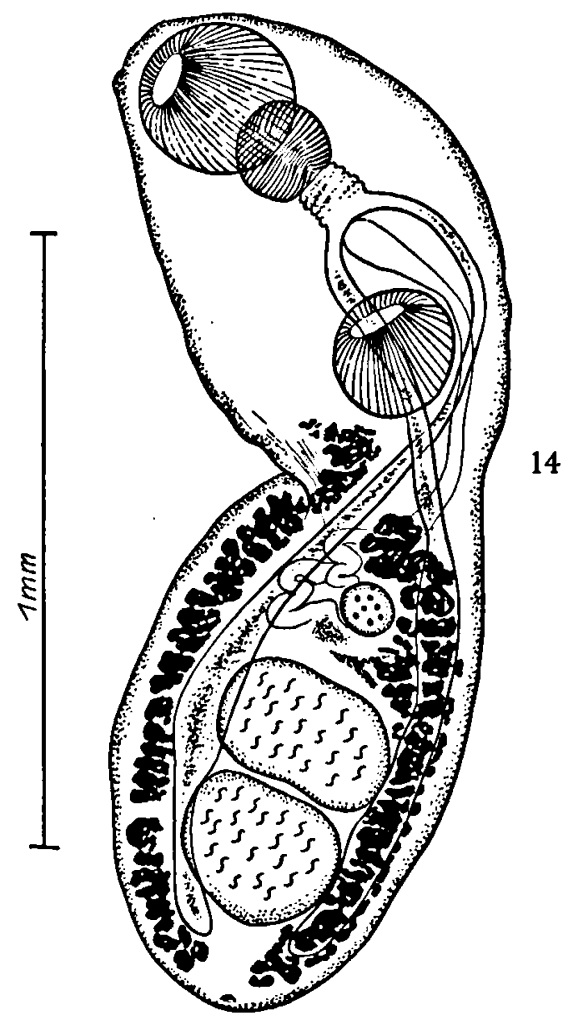

Abb. 14. Psilostomum brevicolle aus Somateria mollissima. Orig. ZIEGER.

II. Paramphistomatida (Poche, 1926) stat. et char. emend.

Notocotylata (Poche, 1926) Skrjabin \& SUL'c, 1933 Notocotylidae LüHE, 1909

TABelle 11. Catatropis verrucosa, Maße von 6 Exemplaren in mm.

\begin{tabular}{|l|l||l|l|l|l|l|}
\hline \multicolumn{1}{|c|}{ Wirt } & Bucephala & \multicolumn{5}{c|}{ Somateria } \\
\hline Körperlänge & 1,9 & 2,2 & 4,1 & 3 & 3 & 2,1 \\
max. Körperbreite & 0,792 & 0,8 & 1,2 & 1 & 1,4 & 0,7 \\
$\begin{array}{l}\text { Mundsaugnapf } \\
\text { Länge }\end{array}$ & 0,072 & 0,147 & 0,154 & 0,147 & 0,139 & 0,139 \\
$\quad$ Breite & 0,097 & 0,147 & 0,220 & 0,169 & 0,206 & 0,154 \\
$\begin{array}{l}\text { Cirrusbeutel- } \\
\quad \text { Länge }\end{array}$ & 0,602 & 0,990 & 1,246 & 1,173 & 1,114 & 0,990 \\
$\begin{array}{l}\text { Metraterm- } \\
\text { Länge }\end{array}$ & 0,477 & 0,806 & 1,114 & 0,990 & 0,880 & 0,792 \\
\hline
\end{tabular}

15. Catatropis verrucosa (FröLICH, 1789)

Wirt/Herkunft: 1 Somateria mollissima (L.), 1 \% Bucephala clangula (L.) / eingeliefert von der Insel Hiddensee, Sektion am 6.2.1963 (Somateria) und 15.1.1963 (Bucephala).
Lokalisation: Blinddärme (Somateria), Dünndarm (Bucephala).

Präparat-Nr.: kT 15/19-25 (51 Exemplare aus Somateria), kT 14/32 (1 Exemplar aus Bucephala).

Beschreibung (vgl. Tabelle 11 und Abb. 15): Cuticula mit kleinen Schuppen besetzt; Körper $2-4,2 \mathrm{~mm}$ lang, maximale Breite 0,8 - 1,5 mm; Cirrus mit war- 

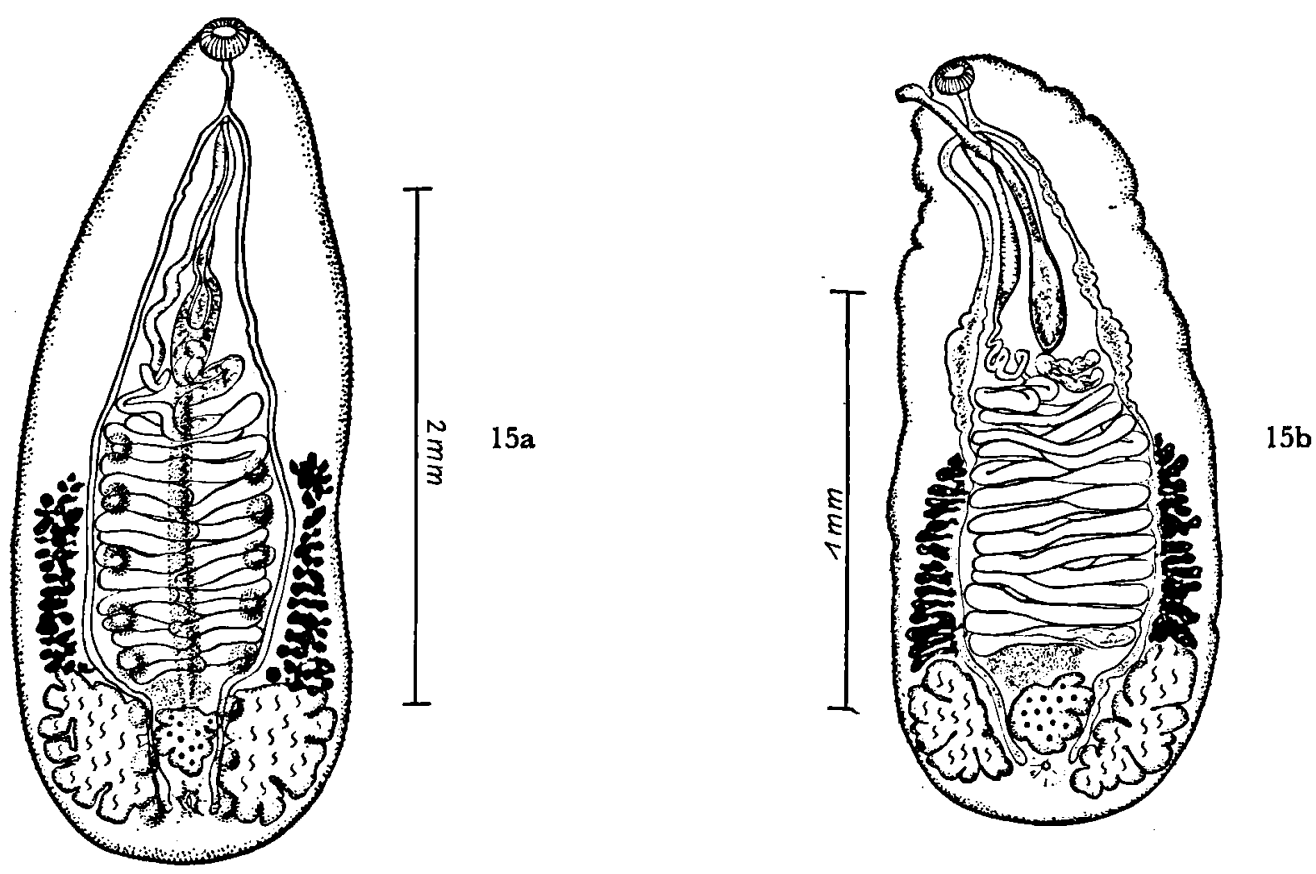

Abb. 15. Catatropis verrucosa. a aus Somateria mollissima, b aus Bucephala clangula. Orig. ZIEGER.

zenartigen Papillen; meist 8 Ventraldrüsen jederseits; Eigröße $16-19 \times 9-11 \mu \mathrm{m}$.

Bemerkungen. ReImer (1962) stellte diese Art bei 9 verschiedenen Anatidae-Arten auf Hiddensee fest. Dieser Liste können nun noch Somateria mollissima und Bucephala clangula hinzugefügt werden.

16. Paramonostomum alveatum (MEHIS in Creplin, 1846)

Wirt/Herkunft: 1 \& Somateria mollissima (L.) / eingeliefert von der Insel Hiddensee, Sektion am 6.2. 1963.

Lokalisation: Mittlerer Dünndarm und Enddarm. Präparat-Nr.: kT 15/26, 28-30 (10 Exemplare).

Beschreibung (vgl. Tabelle 12 und Abb. 16): Cuticula im Vorderkörper sehr fein bestachelt; Körper rundlich-elliptisch, 0,536 - 1,063 mm lang, maximale Breite 0,404-0,661 mm; Eigröße 18 - $21 \times 9-11$ $\mu \mathrm{m}$.

Bemerkungen. Reimer (1962) stellte diese Art bei 5 verschiedenen Anatidae-Arten auf Hiddensee fest. $\mathrm{Zu}$ dieser Liste kommt nun noch Somateria mollissima.
TABelle 12. Paramonostomum alveatum aus Somateria mollissima, Maße von 5 Exemplaren in $\mathrm{mm}$.

\begin{tabular}{|l|l|l|l|l|l|}
\hline Körperlänge & 0,916 & 1,063 & 0,755 & 0,697 & 0,719 \\
max. Körperbreite & 0,631 & 0,661 & 0,617 & 0,558 & 0,514 \\
$\begin{array}{l}\text { Mundsaugnapf } \\
\text { Länge }\end{array}$ & 0,079 & 0,076 & 0,079 & 0,079 & 0,069 \\
$\begin{array}{l}\text { Breite } \\
\text { Cirrusbeutel- } \\
\text { Breite }\end{array}$ & 0,090 & 0,079 & 0,086 & 0,093 & 0,069 \\
\hline
\end{tabular}

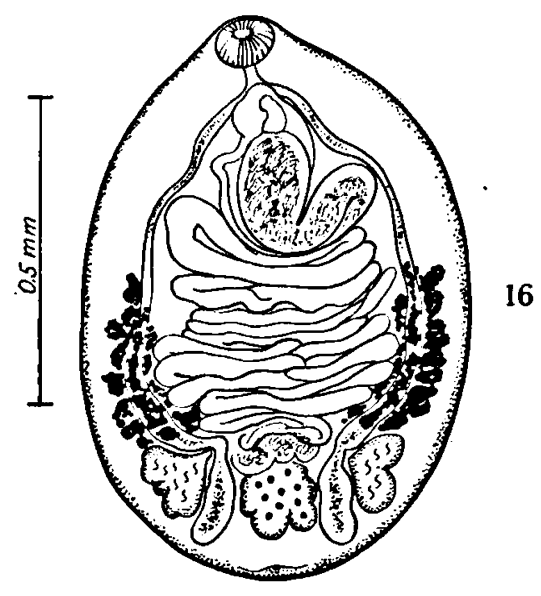

Abb. 16. Paramonostomum alveatum aus Somateria mol lissima. Orig. ZIEGER. 
III. Cyclocoelida (LA RuE, 1957) stat. et nom. emend. Cyclocoelidae Kossack, 1911

\section{Cyclocoelum (Cyclocoelum) mutabile (ZEDER, 1800)}

Wirt/Herkunft: 1 ㅇ Gallinula chloropus (L.) / aus der Umgebung von Berlin eingeliefert, Sektion am 13.2.1963.

Lokalisation: Leibeshöhle (Brustbereich).

Präparat-Nr.: kT 15/1 (1 Exemplar).

Beschreibung (vgl. Abb. 17): Cuticula von kleinen Papillen durchsetzt; Körper hinten abgerundet, vorn verschmälert, $15,3 \mathrm{~mm}$ lang bei einer maximalen Breite von 7,3 mm; Mundsaugnapf nur sehr schwach entwickelt, $0,345 \times 0,499 \mathrm{~mm}$ groß; Pharynx 0,661 $\mathrm{mm}$ lang und $0,683 \mathrm{~mm}$ breit; vorderer Testis $0,587 \times 0,514 \mathrm{~mm}$ groß, hinterer Testis queroval, $0,536 \times 1,041 \mathrm{~mm}$ groß; Ovarium $0,528 \mathrm{~mm}$ im Durchmesser; Genitalporus vor der Mitte des Pharynx; Eigröße $100-110 \times 53-69 \mu \mathrm{m}$.

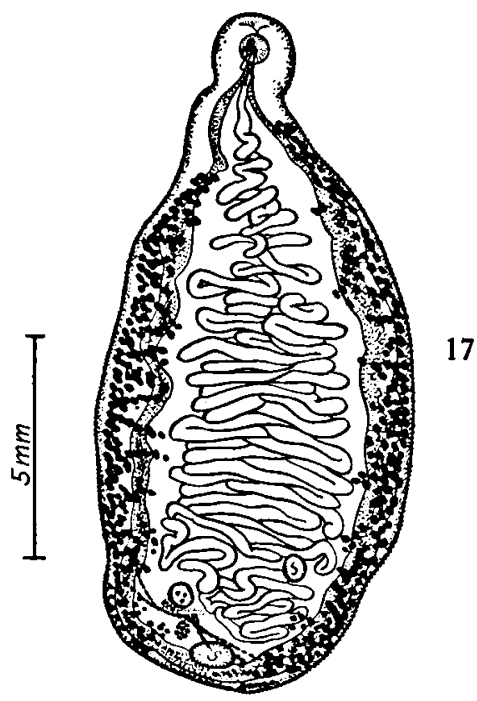

Abb. 17. Cyclocoelum mutabile aus Gallinula chloropus. Orig. ZIEGER.

Bemerkungen. $\mathrm{Zu}$ dieser Art und ihren Synonymen siehe Dubors (1959) sowie auch BezuBIK (1956a), SUlgostowsKa (1958, 1960b) und Macko (1961/62).

\section{LITERATUR}

BaEr, J. G., \& CH. Joyeux, 1961: Classe des trématodes, in Grassé, P.-P.: Traité de zoologie, Tome IV, Premier fascicule, 561-692. Paris.

BAŠxIRovA, E., JA., 1941: Eschinostomatidy ptic SSSR i obzor ciklov ich razvitija. Trudy baškir. n.-i. vet. St. 3 , 243-300.

BEZUBIK, B., 1956a: Materials to the helminthofauna of aquatic birds of Poland. Acta parasitol. polon. 4 (2), 59-88.

-1956b: The helminthfauna of wild ducks (subfam. Anatinae). Acta parasitol. polon. 4 (10), 407-510.

Bychovskaja-Pavlovskaja, I. E., 1962: Die Vogeltrematoden der Fauna der UdSSR (russisch). Moskau-Leningrad, Verl. Akad. Wiss. UdSSR, 407 pp.

DiETz, E., 1910: Die Echinostomiden der Vögel. Zool. Jb. Supp. 12 (3), 265-512, Taf. 10-15 (Festschr. MAX Braun 60. Geb.-Tg.).

Dubors, G., 1959: Revision des Cyclocoelidae Kossacx 1911 (Trematoda). Rev. suisse Zool. 66 (1), 67-147.

Dubors, G., \& J. Maron, 1959: Etude de quelques trématodes nord-américains (avec note sur la position systématique de Parorchis Nicolr 1907) suivie d'une revision des genres Galactosomum Looss 1899 et Ochetosoma Braun 1901. Bull. Soc. neuchâtel. Sci. natur. 82, 191229.

GuptA, R., 1962: On Ignavia breviovarica sp.nov., from the purple heron, Ardea purpurea (LinNaEus) with a note on the validity of Brijicola caballeroi PANDE, 1960 (Trematoda: Echinostomatidae). Rev. biol. trop. Univ. Costa Rica 10 (1), 99-109.
KURAŠvII, B. E., 1957: Die Helminthen der jagdbaren Vögel Grusiniens in faunistischer und ökologischer Hinsicht (russisch). Moskau, Verl. Akad. Wiss. UdSSR, $434 \mathrm{pp}$.

LA RuE, G. R., 1957: The Classification of Digenetic Trematoda: A Review and a New System. Exper. Parasitol. 6, 306-349.

Lumsden, R. D., \& J. A. ZischKe, 1963: Studies on the trematodes of Louisiana birds. Z. Parasitenk. 22, 316366.

MACro, J. K.: K helmintofaune potápkovitých vtákov na východnom Slovensku. Csl. Parasitologie 6 (1), 127-158.

- 1960a: K faune plathelmintov volavky purpurovej (Ardea purpurea L.). Biológia (Bratislava) 15 (8), 608-612.

- 1960b: K faune plathelmintov volavky popolavej (Ardea cinerea $L_{\text {L.) }}$ na východnom Slovensku. Acta Mus. Slovaciae Reg. orient. (Košice), A - Hist. natur. 1, 91-109.

- 1961/62: Plathelminthen und ihre Erforschung bei den am häufigsten vorkommenden freilebenden Vögeln in der Ostslowakei (slowakisch m. russ., engl. u. dtsch. Res.). Acta Mus. Slovaciae Reg. orient. (Kosiče), A Hist. natur. 2-3, 129-154.

MACKO, J. K., \& K. ODEning, 1963: Uber Echinoparyphium recurvatum (v. Linstow, 1873) ? aus Rallus aquaticus L. Ref. in Mber. dtsch. Akad. Wiss. Berlin 5 (2), 134-135. - Studia helminthologica (CSSR) (2) i. Dr. MENDHEn, H., 1940: Beiträge zur Systematik und Biologie der Familie Echinostomatidae (Trematoda). Nova Acta leopoldina (Halle/Saale) N.F. 8 (54), 489-588.

- 1943: Beiträge zur Systematik und Biologie der Familie 
Echinostomatidae. Arch. Naturgesch. N. F. 12 (2), 175-302.

OdenING, K., 1960: Zur Großeinteilung der digenetischen Trematoden. Z. Parasitenk. 20, 170-174.

- 1961a: Zur Kenntnis der Trematodenfauna einiger Vogelarten. Mitt. zool. Mus. Berlin 37 (1), 125-146.

- 1981b: Historische und moderne Gesichtspunkte beim Aufbau eines natürlichen Systems der digenetischen Trematoden. Biol. Beitr. (Jena) 1 (1), 73-90.

- 1962a: Echinocollum vietnamense n. gen. n. sp. (Trematoda, Echinostomatoidea: Echinocollidae n. fam.) aus Seidenreihern der Demokratischen Republik Vietnam. Mber. dtsch. Akad. Wiss. Berlin 3 (11/12), 696-703. 1962b: Trematoden aus indischen Vögeln des Berliner Tierparks. Z. Parasitenk. 21, 381-425.

- 1962c: Trematoden aus einheimischen Vögeln des Berliner Tierparks und der Umgebung von Berlin (Vorläufige Mitteilung). Mber. dtsch. Akad. Wiss. Berlin 4 (3-4), 228-234.

- 1962d: Bemerkungen zum Exkretionssystem dreier echinostomer Cercarien sowie zur Identität der Gattungen Neoacanthoparyphium YamaguTI und Allopetasiger YamaguTI (Trematoda: Echinostomatidae). Z. Parasitenk. 21, 521-534.

- 1962e: Trematoden aus einheimischen Vögeln des Berliner Tierparks und der Umgebung von Berlin. Biol. Zbl. 81 (4), 419-468.

- 1962f: Was ist Cercaria spinifera LA VALETte? Bemerkungen zur artlichen Identität und Biologie einiger echinostomer Cercarien. Symposium über an Wasserbedingungen gebundene Helminthen, 5 pp. Prag.

- im Druck a: Zur Trematodenfauna der einheimischen Lappentaucher und des BleBhuhns. Abh. Ber. Naturk. Vorgesch. Magdeburg.

- im Druck b: Trematoden aus einheimischen Stockenten und Lachmöwen. Zool. Anz.

- im Druck c: Einige Trematoden von aus der Sowjetunion importierten Vögeln des Berliner Tierparks. Zool. Abh. Ber. Mus. Tierk. Dresden 26 (12) (1963).

- im Druck d: Strigeida aus Vögeln des Berliner Tierparks. Angew. Parasitol. (Jena) $4(3,4)$, (1963).

ODHNER, T., 1910: Nordostafrikanische Trematoden, größtenteils vom Weißen Nil (von der Schwedischen Zoologischen Expedition gesammelt) I. Fascioliden. Results swed. zool. Exped. Egypt White Nile (4), 1-166, 6 Taf. Uppsala.
Prudhoe, S., 1960: On two new Parasitic Worms from Brazil. Libro Homen. Dr. E. Cabaluero y C. (México), 415-421.

Reamer, L., 1962: Die digenetischen Trematoden der Wasservögel der Insel Hiddensee und ihre Larvalstadien aus den die Insel umgebenden Brackgewässem. Inaug.Diss. Math.-naturw. Fak. Univ. Greifswald, 128 pp.

- 1963: Curtuteria numenii nov. gen. nov. spec. aus Numenius phaeopus (L.) (Trematoda, Digenea, Echinostomatidae, Himasthlinae). Z. Parasitenk. 23, 249-252.

Šign, A. A., 1956: Uber die Selbständigkeit der Gattung Episthmium (LÜHE, 1909) in Verbindung mit der Beschreibung einer neuen Art Ep. colymbi nov. sp. aus dem Haubentaucher (russisch). Trudy biol. Stancii "Borok" 2, 327-334.

Smmi, S. S., 1958: Studies on the trematode parasites of reptiles found in Hyderabad State. Z. Parasitenk. 18, 161-218.

- 1962: A note on a new trematode subfamily Singhiatreminae. Current Science 31,68 .

SkrJABn, K. I., 1947, 1950, 1953, 1956: Die Trematoden der Tiere und des Menschen. Grundlagen der Trematodologie (russisch). 1, 4, 8, 12. Moskau, Verl. Akad. Wiss. UdSSR.

SulcostowsKA, T., 1958: Flukes of birds of Družno Lake (Parasitofauna of the biocoenosis of Družno Lake part III). Acta parasitol. polon. 6 (3), 111-142.

- 1960a: Intestinal trematodes of birds of mesotrophic lakes: Gołdapiwo and Mamry Pólnocne. Acta parasitol. polon 8 (6), 85-114.

- 1960b: Extra-intestinal trematodes in birds of the mesotrophic lakes: Gołdawipo and Mamry Połnocne. Acta parasitol. polon. 8 (32), 471-492.

Wrichr, C. A., 1957: Two Kidney-Flukes from Sudanese Birds, with a Description of a New Species. J. Helminthol. 31 (4), 229-238.

YamacuT, S., 1958: Systema Helminthum. Vol. I. The digenetic Trematodes of Vertebrates. Part 1, II. New York/London, 1575 pp.

YEH, L.-S., 1954: On a New Trematode Allechinostomum renale sp. nov. (Trematoda: Echinostomatidae), from Pelecanus erythrorhynchos. J. Helminthol. 28 (3/4), 159-164. 